\title{
INTRODUCTION TO HYPERCONVEX SPACES
}

\author{
R. ESPÍNOLA AND M. A. KHAMSI
}

\section{Contents}

$\begin{array}{lr}\text { 1. Preface } & 1 \\ \text { 2. Introduction and basic definitions } & 2 \\ \text { 3. Some basic properties of Hyperconvex spaces } & 4 \\ \text { 4. Hyperconvexity, Injectivity and Retraction } & 8 \\ \text { 5. More on Hyperconvex spaces } & 15 \\ \text { 6. Fixed point property and Hyperconvexity } & 20 \\ \text { 7. Topological fixed point theorems and Hyperconvexity } & 23 \\ \text { 8. Isbell's Hyperconvex Hull } & 27 \\ \text { 9. Set-valued mappings in Hyperconvex spaces } & 30 \\ \text { 10. The KKM theory in Hyperconvex spaces } & 35 \\ \text { 11. Lambda-Hyperconvexity } & 38 \\ \text { References } & 40\end{array}$

\section{PREFACE}

The notion of hyperconvexity is due to Aronszajn and Panitchpakdi [1] (1956) who proved that a hyperconvex space is a nonexpansive absolute retract, i.e. it is a nonexpansive retract of any metric space in which it is isometrically embedded. The corresponding linear theory is well developed and associated with the names of Gleason, Goodner, Kelley and Nachbin (see for instance $[19,29,42,46])$. The nonlinear theory is still developing. The recent interest into these spaces goes back to the results of Sine [54] and Soardi [57] who proved independently that fixed point property for nonexpansive mappings holds in bounded hyperconvex spaces. Since then many interesting results have been shown to hold in hyperconvex spaces.

Recall also that Jawhari, Misane and Pouzet [27] were able to show that Sine and Soardi's fixed point theorem is equivalent to the classical Tarski's fixed point theorem in complete ordered sets. This happens via the notion of generalized metric spaces. Therefore, the notion of hyperconvexity should be understood and appreciated in a more abstract formulation. It is not our purpose, however, to study hyperconvex spaces from this more general point of view, interested readers may consult the references [22, 27, 28, 38, 59].

Along this chapter we will describe and study some of the most characteristic properties that hyperconvex spaces enjoy. In opposition to the lack of linearity hyperconvexity provides us with a really rich metric structure that leads to a collection of surprising and beautiful results related to different branches of mathematics as, for instance, topology, graph theory, multivalued analysis, fixed point theory,... It is our aim to present some of these results about hyperconvexity emphasizing their relation to fixed point theory. 
This chapter has been divided into eleven sections. We begin with basic definitions and properties which will lead us to a first approach to the relation between hyperconvexity and the Hahn-Banach theorem. In Section 3 we continue with more general properties on hyperconvexity, we will learn some fundamental facts of the geometry of hyperconvex spaces, facts such as that every hyperconvex space is complete or relevant properties verified by their Chebyshev elements. In this section we will also distinguish among four different important subclasses of hyperconvex subsets of a hyperconvex space, namely hyperconvex, admissible, externally and weakly externally hyperconvex subsets. These classes of sets will be of great importance all along our treatment and more particularly when fixed point results will be stated. In Section 4 we relate hyperconvexity to injectivity and absolute retracts. We will see the role that these latter concepts played in the motivations of hyperconvexity to finish with more recent and subtle results on the existence of retractions and $\varepsilon$-constant nonexpansive retractions. Section 5 will be devoted to the study of deeper facts of the geometry of hyperconvex spaces, we begin by studying the bad properties of these spaces with respect to the intersection, stating that hyperconvex subsets of a hyperconvex spaces do not define a closed (under the intersection of sets) class of sets. This is in part corrected by a very celebrated result due to Baillon in [3] on intersection of hyperconvex sets that allows to define the concept of "hyperconvex hull" as that one of "injective hull" given by Isbell in [24]. This section is finished with the definition of the noncompactness measures of Hausdorff and Kuratowski as well as their properties in hyperconvex spaces.

We abandon generalities on hyperconvexity in Section 6 to study some more recent and particular properties that will lead us to deep fixed point results. The existence of fixed point for nonexpansive mappings is studied as well as similar results on family of commuting mappings. This section is closed by a non-elsewhere published result on asymptotically nonexpansive mappings. Section 7 deals with another kind of fixed point theorems, those in which compactness conditions are considered. We study how different results stated in linear spaces are still true in a hyperconvex setting. Section 8 is completely devoted to the study of the "injective hull" of Isbell and extremal functions, this concept is one of the most interesting and intriguing ones in hyperconvex metric spaces and well deserves a whole section for its better understanding. Another very important characteristic of hyperconvex spaces is studied in Section 9, in this section we focus on multivalued mappings and state a surprising result on selection of multivalued mappings that implies different results on fixed point theory and existence of nonexpansive selections of the metric projection. The study on multivalued mappings will be completed in Section 10 where the KKM principle is adapted for hyperconvex spaces and new versions of classical results on fixed point theory are obtained. The concept of "lambda hyperconvexity" is studied in the last section of the chapter, Section 11. This is a recently introduced [34] idea which may be understood as an extension of the geometrical definition of hyperconvexity inspired in former studies due to Grnbaum [20].

Finally we want to point out that the material we present here goes from classical to very recent facts that will lead the reader to an updated knowledge about fixed point results on hyperconvex spaces. The reader will find, however, a large collection of items at the end of the chapter from which it is possible to continue the study of facts and related subjects that were not treated in detail in this chapter.

\section{INTRODUCTION AND BASIC DEFINITIONS}

No doubt that Hahn-Banach theorem played a major role in functional analysis. In fact, it is quite impossible to think of Banach spaces without this theorem. So it was clear from the beginning that an extension of this theorem to metric spaces was to be found. The first to study 
this question were Aronszajn and Panitchpakdi in [1]. Their investigation led to the discovery of hyperconvex metric spaces. In order to appreciate their findings, one needs to remember the proof of Hahn-Banach theorem.

Theorem 2.1. Let $X$ be a real vector space, $Y$ be a linear subspace of $X$, and $\rho$ a seminorm on $X$. Let $f$ be a linear functional defined on $Y$ such that $f(y) \leq \rho(y)$, for all $y \in Y$. Then there exists a linear functional $g$ defined on $X$, which is an extension of $f(i . e . g(y)=f(y)$, for all $y \in Y)$, which satisfies $g(x) \leq \rho(x)$, for all $x \in X$.

Proof. The main argument behind the proof of this theorem is the structure of the real line $\overline{\mathbb{R}}$. Indeed, via Hausdorff maximality principle, it is enough to extend $f$ to $Y+\mathbb{R} \cdot x_{0}$, where $x_{0} \in X-Y$. So we need to find $g\left(x_{0}\right)$ such that

$$
\left\{\begin{array}{l}
g\left(y+\alpha x_{0}\right)=g(y)+\alpha g\left(x_{0}\right)=f(y)+\alpha g\left(x_{0}\right) \\
g\left(y+\alpha x_{0}\right) \leq \rho\left(y+\alpha x_{0}\right)
\end{array}\right.
$$

for any real number $\alpha \in \mathbb{R}$ and any $y \in Y$. Since $\rho$ is a seminorm and $f$ is a linear functional, we may assume $\alpha= \pm 1$. This means that what we need to find is a number $A$ (which eventually will be equal to $\left.g\left(x_{0}\right)\right)$, such that $f(y) \pm A \leq \rho\left(y \pm x_{0}\right)$, which translates to $f(y)-\rho\left(y-x_{0}\right) \leq$ $A \leq \rho\left(y^{*}+x_{0}\right)-f\left(y^{*}\right)$ for any $y, y^{*} \in Y$. In other words, we must have

$$
\bigcap_{y, y^{*} \in Y}\left[f(y)-\rho\left(y-x_{0}\right), \rho\left(y^{*}+x_{0}\right)-f\left(y^{*}\right)\right] \neq \emptyset .
$$

Set $I_{y, y^{*}}=\left[f(y)-\rho\left(y-x_{0}\right), \rho\left(y^{*}+x_{0}\right)-f\left(y^{*}\right)\right]$, for $y, y^{*} \in Y$. Then using the linearity of $f$ and the seminorm behavior of $\rho$, it is easy to check that for any $y_{1}, y_{2}, y_{1}^{*}$, and $y_{2}^{*}$ in $Y$, $I_{y_{1}, y_{1}^{*}} \cap I_{y_{1}, y_{1}^{*}} \neq \emptyset$. The proof will be complete if we use the following well-known fundamental property of the real line $\mathbb{R}$ :

"If $\left\{I_{\alpha}\right\}_{\alpha \in \Gamma}$ is a collection of intervals such that $I_{\alpha} \cap I_{\beta} \neq \emptyset$, for any $\alpha, \beta \in \Gamma$, then we have $\bigcap_{\alpha \in \Gamma} I_{\alpha} \neq \emptyset "$

It is this property that is at the heart of the new concept discovered by Aronszajn and Panitchpakdi. Note that an interval may also be seen on the real line as a closed ball. Indeed, the interval $[a, b]$ is also the closed ball centered at $(a+b) / 2$ with radius $r=(b-a) / 2$, i.e. $[a, b]=B\left(\frac{a+b}{2}, \frac{b-a}{2}\right)$.

So the above intersection property may also be seen as a ball intersection property. This is quite interesting since in metric spaces it is natural to talk about balls. But keep in mind that in ordered sets for example, intervals are more natural than balls.

Throughout this chapter, the balls referred to are closed. Therefore we will omit the word closed.

Remark 2.2. Let $M$ be a metric space. Using the triangle inequality, we have $B\left(x_{1}, r_{1}\right) \cap$ $B\left(x_{2}, r_{2}\right) \neq \emptyset$ implies $d\left(x_{1}, x_{2}\right) \leq r_{1}+r_{2}$ for any $x_{1}, x_{2} \in M$ and positive numbers $r_{1}, r_{2}$. The converse is true on the real line and corresponds to the Menger convexity in metric spaces.

Definition 2.3. Let $M$ be a metric space. We say that $M$ is metrically convex if for any points $x, y \in M$ and positive numbers $\alpha$ and $\beta$ such that $d(x, y) \leq \alpha+\beta$, there exists $z \in M$ such that $d(x, z) \leq \alpha$ and $d(z, y) \leq \beta$, or equivalently $z \in B(x, \alpha) \cap B(y, \beta)$. 
Therefore, $M$ is metrically convex if $B(x, \alpha) \cap B(y, \beta) \neq \emptyset$ if and only if $d(x, y) \leq \alpha+\beta$ for any points $x, y \in M$ and positive numbers $\alpha$ and $\beta$.

Remark 2.4. Note that some authors define metric convexity slightly differently. Indeed, $(M, d)$ is metrically convex if and only if for any points $x, y \in M$ and any number $\alpha \in[0,1]$, there exists $z \in M$ such that $d(x, z)=\alpha d(x, y)$ and $d(y, z)=(1-\alpha) d(x, y)$. This definition is hard to extend to general structures since it uses the multiplication operation, which is, for example, hard to define in discrete sets.

The above discussion shows that the Hahn-Banach extension theorem is closely related to an intersection property of the closed balls combined with some kind of metric convexity. The concept of hyperconvexity introduced by Aronszajn and Panitchpakdi captured these ideas.

Definition 2.5. The metric space $M$ is said to be hyperconvex if $\bigcap_{\alpha \in \Gamma} B\left(x_{\alpha}, r_{\alpha}\right) \neq \emptyset$ for any collection of points $\left\{x_{\alpha}\right\}_{\alpha \in \Gamma}$ in $M$ and positive numbers $\left\{r_{\alpha}\right\}_{\alpha \in \Gamma}$ such that $d\left(x_{\alpha}, x_{\beta}\right) \leq r_{\alpha}+r_{\beta}$ for any $\alpha$ and $\beta$ in $\Gamma$.

\section{Some BAsic properties of Hyperconvex spaCes}

Clearly from the previous section, the real line $\mathbb{R}$ is hyperconvex. In fact, we can easily prove that the infinite dimensional Banach space $l_{\infty}$ is hyperconvex. One way to see that is to use the following result.

Theorem 3.1. Let $\left(M_{\alpha}, d_{\alpha}\right)_{\alpha \in \Gamma}$ be a collection of hyperconvex metric spaces. Consider the product space $\mathcal{M}=\prod_{\alpha \in \Gamma} M_{\alpha}$. Fix $a=\left(a_{\alpha}\right) \in \mathcal{M}$ and consider the subset $M$ of $\mathcal{M}$ defined by

$$
M=\left\{\left(x_{\alpha}\right) \in \mathcal{M} ; \sup _{\alpha \in \Gamma} d_{\alpha}\left(x_{\alpha}, a_{\alpha}\right)<\infty\right\} .
$$

Then $\left(M, d_{\infty}\right)$ is a hyperconvex metric space where $d_{\infty}$ is defined by

$$
d_{\infty}\left(\left(x_{\alpha}\right),\left(y_{\alpha}\right)\right)=\sup _{\alpha \in \Gamma} d_{\alpha}\left(x_{\alpha}, y_{\alpha}\right)
$$

for any $\left(x_{\alpha}\right),\left(y_{\alpha}\right) \in M$.

This theorem is a structural result. Its proof is an easy consequence from the fact that for any ball $B\left(\left(x_{\alpha}\right), r\right)$ in $M$, we have $B\left(\left(x_{\alpha}\right), r\right)=\prod_{\alpha \in \Gamma} B_{\alpha}\left(x_{\alpha}, r\right)$ where $B_{\alpha}\left(x_{\alpha}, r\right)$ is the ball centered at $x_{\alpha}$ with radius $r$ in $M_{\alpha}$.

We will see later on that any metric space may be embedded in a "small" hyperconvex metric space. That construction, discovered by Isbell, is not immediate and hard to grasp. But for many cases, it is worth to know that any metric space may be embedded isometrically into a hyperconvex metric space. This is easy to see. Indeed, let $(M, d)$ be a metric space. Set

$$
l_{\infty}(M)=\left\{\left(x_{m}\right)_{m \in M} \in \mathbb{R}^{M} ; \sup _{m \in M}\left|x_{m}\right|<\infty\right\} .
$$

On $l_{\infty}(M)$ define the distance $d_{\infty}$ by $d_{\infty}\left(\left(x_{m}\right),\left(y_{m}\right)\right)=\sup _{m \in M}\left|x_{m}-y_{m}\right|$. The metric space $\left(l_{\infty}(M), d_{\infty}\right)$ is hyperconvex. To see that $M$ embeds isometrically into $l_{\infty}(M)$, fix $a \in M$ and consider the map $I: M \rightarrow l_{\infty}(M)$ defined by $I(b)=(d(b, m)-d(a, m))_{m \in M}$ for any $b \in M$. It 
is easy to check that $d_{\infty}(I(b), I(c))=d(b, c)$ for any $b, c \in M$.

Next we discuss completeness of hyperconvex metric spaces. In fact, a weaker version of the binary-ball intersection property is needed to insure the completeness of the metric space. Indeed, we will say that the metric space $M$ has the ball intersection property (BIP in short) if $\bigcap_{\alpha \in \Gamma} B_{\alpha} \neq \emptyset$ for any collection of balls $\left(B_{\alpha}\right)_{\alpha \in \Gamma}$ such that $\bigcap_{\alpha \in \Gamma_{f}} B_{\alpha} \neq \emptyset$, for any finite subset $\Gamma_{f} \subset \Gamma$.

Proposition 3.2. Any metric space $M$ which has the ball intersection property is complete. In particular any hyperconvex metric space is complete.

$\underline{\text { Proof. }}$ Let $\left(x_{n}\right)$ be a Cauchy sequence in $M$. For any $n \geq 1$, set $r_{n}=\sup _{m \geq n} d\left(x_{n}, x_{m}\right)$. Consider the collection of balls $\left(B\left(x_{n}, r_{n}\right)\right)_{n \geq 1}$. Since for $m \geq n$ we have $d\left(x_{n}, x_{m}\right) \leq r_{n}$, then

$$
x_{n_{k}} \in B\left(x_{n_{1}}, r_{n_{1}}\right) \cap B\left(x_{n_{2}}, r_{n_{2}}\right) \cap \cdots \cap B\left(x_{n_{k}}, r_{n_{k}}\right)
$$

for any $n_{1}<n_{2}<\cdots<n_{k}$, but $M$ has the ball intersection property so we may conclude that $\bigcap_{n \geq 1} B\left(x_{n}, r_{n}\right) \neq \emptyset$. Now, since $\left(x_{n}\right)$ is a Cauchy sequence, $\lim _{n \rightarrow \infty} r_{n}=0$ and so the intersection $\bigcap_{n \geq 1}^{n \geq 1} B\left(x_{n}, r_{n}\right)$ is reduced to one point $z$ which is the limit of the sequence $\left(x_{n}\right)$.

We have just seen a property about intersection of balls rather than about hyperconvexity. Ball intersection properties have been extensively studied in connection with different problems as geometrical properties of Banach spaces or extension of mappings. Interested readers may find more on this interesting subject in $[6,23,43,45]$.

At this point we introduce some notation which will be used throughout the remainder of this work. For a subset $A$ of a metric space $M$, set:

$$
\begin{array}{ll}
r_{x}(A) & =\sup \{d(x, y): y \in A\}, \quad x \in M ; \\
r(A) & =\inf \left\{r_{x}(A): x \in M\right\} ; \\
R(A) & =\inf \left\{r_{x}(A): x \in A\right\} ; \\
\operatorname{diam}(A) & =\sup \{d(x, y): x, y \in A\} ; \\
C(A) & =\left\{x \in M: r_{x}(A)=r(A)\right\} ; \\
C_{A}(A) & =\left\{x \in A: r_{x}(A)=r(A)\right\} ; \\
\operatorname{cov}(A) & =\bigcap\{B: B \text { is a ball and } B \supseteq A\} .
\end{array}
$$

$r(A)$ is called the radius of $A$ (relative to $M$ ), $\operatorname{diam}(A)$ is called the diameter of $A, R(A)$ is called the Chebyshev radius of $A, C(A)$ is called the center of $A$ (in $M), C_{A}(A)$ is called the Chebyshev center of $A$, and $\operatorname{cov}(A)$ is called the cover of $A$.

We now prove a technical lemma.

Lemma 3.3. Suppose $A$ is a bounded subset of a hyperconvex metric space $M$. Then:

1. $\operatorname{cov}(A)=\bigcap\left\{B\left(x, r_{x}(A)\right): x \in M\right\}$.

2. $r_{x}(\operatorname{cov}(A))=r_{x}(A)$, for any $x \in M$.

3. $r(\operatorname{cov}(A))=r(A)$.

4. $r(A)=\frac{1}{2} \operatorname{diam}(A)$.

5. $\operatorname{diam}(\operatorname{cov}(A))=\operatorname{diam}(A)$.

6. If $A=\operatorname{cov}(A)$, then $r(A)=R(A)$. In particular we have $R(A)=\frac{1}{2} \operatorname{diam}(A)$. 
Proof. 1. Since $B\left(x, r_{x}(A)\right)$ contains $A$ for each $x \in M$ it must be the case that

$$
\operatorname{cov}(A) \subseteq \bigcap\left\{B\left(x, r_{x}(A)\right): x \in M\right\} .
$$

On the other hand, if $A \subseteq B(x, r)$ then $r_{x}(A) \leq r$ so $B\left(x, r_{x}(A)\right) \subseteq B(x, r)$. Hence

$$
\bigcap\left\{B\left(z, r_{z}(A)\right): z \in M\right\} \subseteq B(x, r) \text {. }
$$

This clearly implies

$$
\operatorname{cov}(A)=\bigcap\left\{B\left(x, r_{x}(A)\right): x \in M\right\}
$$

2. By $1, r_{x}(\operatorname{cov}(A))=\sup \left\{d(x, y): y \in \bigcap_{z \in M} B\left(z, r_{z}(A)\right)\right\}$ so, in particular, $y \in \operatorname{cov}(A)$ implies $y \in B\left(x, r_{x}(A)\right)$ for any $x \in M$. Hence $d(x, y) \leq r_{x}(A)$, which proves $r_{x}(\operatorname{cov}(A)) \leq r_{x}(A)$. The reverse inequality is obvious since $A \subseteq \operatorname{cov}(A)$.

3. This is immediate from the definition of $r$.

4. Let $\delta=\operatorname{diam}(A)$ and consider the family $\left\{B\left(a, \frac{\delta}{2}\right): a \in A\right\}$. If $a, b \in A$ then $d(a, b) \leq$ $\delta=\frac{\delta}{2}+\frac{\delta}{2}$ so by hyperconvexity

$$
\bigcap_{a \in A} B\left(a, \frac{\delta}{2}\right) \neq \emptyset
$$

If $x$ is any point in this intersection then $d(x, a) \leq \frac{\delta}{2}$ so $r_{x}(A) \leq \frac{\delta}{2}$. On the other hand $d(a, b) \leq$ $d(a, z)+d(z, b)$ for any $a, b \in A$ and $z \in M$ so $\delta \leq 2 r_{z}(A)$ from which $\delta \leq 2 r(A)$. Therefore $\delta \leq 2 r(A) \leq 2 r_{x}(A) \leq \delta$ proving $r(A)=\frac{\delta}{2}$.

5. Using 3 and $4, \operatorname{diam}(A)=2 r(A)=2 r(\operatorname{cov}(A))=\operatorname{diam}(\operatorname{cov}(A))$.

6. Note that we always have $\frac{1}{2} \operatorname{diam}(A) \leq r(A) \leq R(A)$. We may write $A=\bigcap_{i \in I} B_{i}$ where $B_{i}$ is a closed ball for any $i \in I$. Now, since $\bigcap_{a \in A} B\left(a, \frac{\delta}{2}\right) \neq \emptyset$ where $\delta=\operatorname{diam}(A)$, it is easy to check that any two balls drawn from the collection

$$
\left\{B_{i}, i \in I\right\} \bigcup\left\{B\left(a, \frac{\delta}{2}\right) ; a \in A\right\}
$$

have nonempty intersection. Recalling now the hyperconvexity of $M$,

$$
\mathcal{C}=A \bigcap\left\{B\left(a, \frac{\delta}{2}\right) ; a \in A\right\}=\bigcap\left\{B_{i} ; i \in I\right\} \bigcap\left\{B\left(a, \frac{\delta}{2}\right) ; a \in A\right\} \neq \emptyset .
$$

Let $x \in \mathcal{C}$. Then $r_{x}(A) \leq \delta / 2$ and therefore $\frac{\delta}{2} \leq r(A) \leq R(A) \leq r_{x}(A) \leq \frac{\delta}{2}$, which clearly implies $r(A)=R(A)=\frac{1}{2} \operatorname{diam}(A)$.

Definition 3.4. Let $M$ be a metric space. By $\mathcal{A}(M)$ we denote the collection of all subsets of $M$ which are intersection of balls, i.e. $\mathcal{A}(M)=\{A \subset M ; A=\operatorname{cov}(A)\}$. The elements of $\mathcal{A}(M)$ are called admissible subsets of $M$. 
It is clear that $\mathcal{A}(M)$ contains all the closed balls of $M$ and is stable by intersection, i.e. the intersection of any collection of elements from $\mathcal{A}(M)$ is also in $\mathcal{A}(M)$. From the above results, for any $A \in \mathcal{A}(M)$, we have

$$
C(A)=\bigcap_{a \in A} B(a, R(A)) \bigcap A \in \mathcal{A}(M) .
$$

Moreover, $\operatorname{diam}(C(A)) \leq \operatorname{diam}(A) / 2$. So we have $A=C(A)$ if and only if $A \in \mathcal{A}(M)$ and $\operatorname{diam}(A)=0$, i.e. $A$ is reduced to one point.

This property and the above studied ones are extremely important when we discuss the fixed point property in hyperconvex metric spaces. In fact, we will show in this chapter that admissible subsets in hyperconvex metric spaces enjoy some nice properties. But admissible subsets will not be the only class of subsets that will be of interest to us, let us introduce three more classes of subsets that will be of great importance in our exposition.

Definition 3.5. A subset $E$ of a metric space $M$ is said to be externally hyperconvex (relative to $M)$ if given any family $\left\{x_{\alpha}\right\}$ of points in $M$ and any family $\left\{r_{\alpha}\right\}$ of real numbers satisfying $d\left(x_{\alpha}, x_{\beta}\right) \leq r_{\alpha}+r_{\beta}$ and $\operatorname{dist}\left(x_{\alpha}, E\right) \leq r_{\alpha}$, where $\operatorname{dist}(x, E)=\inf \{d(x, y): y \in E\}$, then it follows that $\cap B\left(x_{\alpha}, r_{\alpha}\right) \cap E \neq \emptyset$. The class of all the externally hyperconvex subsets of $M$ will be denoted as $\mathcal{E}(M)$.

Definition 3.6. A subset $E$ of a metric space $M$ is said to be weakly externally hyperconvex (relative to $M$ ) if $E$ is externally hyperconvex relative to $E \cup\{z\}$ for each $z \in M$. More precisely, given any family $\left\{x_{\alpha}\right\}$ of points in $M$ all but at most one of which lies in $E$, and any family $\left\{r_{\alpha}\right\}$ of real numbers satisfying $d\left(x_{\alpha}, x_{\beta}\right) \leq r_{\alpha}+r_{\beta}$, with $\operatorname{dist}\left(x_{\alpha}, E\right) \leq r_{\alpha}$ if $x_{\alpha} \notin E$, it follows that $\cap_{\alpha} B\left(x_{\alpha} ; r_{\alpha}\right) \cap E \neq \emptyset$. The class of all the weakly externally hyperconvex subsets of $M$ will be denoted as $\mathcal{W}(M)$.

Additionally, we will denote the class of hyperconvex subsets of a metric space $M$ as $\mathcal{H}(M)$.

We finish this section studying the relation among these classes of sets. In order to do that we need to introduce the concept of proximality.

Definition 3.7. A subset $E$ of a metric space $M$ is said to be proximinal (with respect to $M$ ) if the intersection $E \cap B(x, \operatorname{dist}(x, E))$ is nonempty for each $x \in M$.

Lemma 3.8. If $E$ is either an admissible, externally hyperconvex or weakly externally hyperconvex subset of a hyperconvex metric space $M$, then $E$ is proximinal in $M$.

Proof. We write the proof for the case $E=A$ an admissible subset. Other cases are similar. Set $\overline{A=\bigcap_{i \in I}} B_{i}$. Then for any $\varepsilon>0$, there exists $a_{\varepsilon} \in A$ such that $d\left(x, a_{\varepsilon}\right) \leq \operatorname{dist}(x, A)+\varepsilon$. Clearly this implies $\bigcap_{i \in I} B_{i} \bigcap B(x, \operatorname{dist}(x, A)+\varepsilon) \neq \emptyset$. Since $M$ is hyperconvex, then we must have

$$
A \cap B(x, \operatorname{dist}(x, A))=\bigcap_{i \in I} B_{i} \bigcap\left(\bigcap_{\varepsilon>0} B(x, \operatorname{dist}(x, A)+\varepsilon)\right) \neq \emptyset
$$

which completes the proof that $A$ is proximinal.

Remark 3.9. Notice that we only need $M$ to be hyperconvex in the proved case, i.e. the same result for externally or weak externally hyperconvex subsets does not require hyperconvexity of the 
space $M$. This is essentially due to the fact that for these other subsets the hyperconvex hypothesis is granted by the definition of these sets.

Theorem 3.10. Let $M$ be a hyperconvex metric space, then $\mathcal{A}(M) \subseteq \mathcal{E}(M) \subseteq \mathcal{W}(M) \subseteq \mathcal{H}(M)$.

Proof. $\mathcal{A}(M) \subseteq \mathcal{E}(M)$ : Let $A$ be an admissible subset of $M$ and let $\left\{x_{\alpha}\right\}_{\alpha \in \Gamma}$ be a family of points in $M$ and $\left\{r_{\alpha}\right\}_{\alpha \in \Gamma}$ be family of real numbers satisfying $d\left(x_{\alpha}, x_{\beta}\right) \leq r_{\alpha}+r_{\beta}$ and $\operatorname{dist}\left(x_{\alpha}, A\right) \leq r_{\alpha}$ for any $\alpha, \beta \in \Gamma$. Since $A$ is proximinal, for any $\alpha \in \Gamma$, there exists $a_{\alpha} \in A$ such that $d\left(x_{\alpha}, a_{\alpha}\right)=$ $\operatorname{dist}\left(x_{\alpha}, A\right)$, which gives $A \cap B\left(x_{\alpha}, r_{\alpha}\right) \neq \emptyset$. Since $M$ is hyperconvex, the conditions on both families imply $\cap_{\alpha \in \Gamma} B\left(x_{\alpha}, r_{\alpha}\right) \neq \emptyset$. Since $A$ is admissible and $A \cap B\left(x_{\alpha}, r_{\alpha}\right) \neq \emptyset$ it follows

$$
A \bigcap\left(\bigcap_{\alpha \in \Gamma} B\left(x_{\alpha}, r_{\alpha}\right)\right) \neq \emptyset
$$

which proves the first inclusion.

$\mathcal{E}(M) \subseteq \mathcal{W}(M):$ It follows directly from definition.

$\mathcal{W}(M) \subseteq \mathcal{H}(M)$ : It follows directly from definition.

Remark 3.11. Notice that if in the definition of weak externally hyperconvexity we impose the condition that one of the balls must be out of E, then weakly externally hyperconvex subsets do not have why to be hyperconvex. Think of $\mathbb{R}^{2}$ minus the interior of the unit square with the supremum norm and take $E$ as the border of the unit square. E would be weakly externally hyperconvex under this new definition but not hyperconvex.

Remark 3.12. Additionally, the families $\mathcal{A}(M), \mathcal{E}(M), \mathcal{W}(M)$, and $\mathcal{H}(M)$ do not coincide in general. Let $M$ be the half right real plane endowed with the maximum metric, i.e. $M=\{(x, y) \in$ $\left.\mathbb{R}^{2}: \quad x \geq 0\right\}$ with the maximum metric. Let $E=B((0,-1), 2) \cap M, W=\{(x, x): 0 \leq x \leq 1\}$, and $H=\left\{\left(x, \frac{x}{2}\right): 0 \leq x \leq 1\right\}$ then $E$ is externally hyperconvex in $M$ but not admissible, $W$ is weakly externally hyperconvex in $M$ but not externally hyperconvex, and, finally, $H$ is hyperconvex but not weakly externally hyperconvex.

\section{Hyperconvexity, Injectivity And Retraction}

In this section, we will discuss Aronszajn and Panitchpakdi ideas on how hyperconvexity captures Hahn-Banach extension theorem in metric spaces. Before we state Aronszajn and Panitchpakdi's main result, we recall the definition of nonexpansive mappings.

Definition 4.1. Let $\left(M_{1}, d_{1}\right)$ and $\left(M_{2}, d_{2}\right)$ be metric spaces. A map $T: M_{1} \rightarrow M_{2}$ is said to be Lipschitzian if there exists a constant $k \geq 0$ such that

$$
d_{2}(T(x), T(y)) \leq k d_{1}(x, y)
$$

for any $x, y \in M_{1}$. If $k=1$, the map is called nonexpansive (and contraction if $k<1$ ).

A metric space $M$ is said to be injective if it has the following extension property: Whenever $Y$ is a subspace of $X$ and $f: Y \rightarrow M$ is nonexpansive, then $f$ has a nonexpansive extension $\tilde{f}: X \rightarrow M$. This fact has several nice consequences.

Theorem 4.2. Let $H$ be a metric space. The following statements are equivalent:

(i) $H$ is hyperconvex; 
(ii) $H$ is injective.

Proof. First assume $H$ is hyperconvex. Let $D$ be a metric space and $T: D \rightarrow \mathcal{H}$ be nonexpansive. Let $M$ be a metric space containing $D$ metrically. Consider the following set

$$
\mathcal{C}=\left\{\left(T_{F}, F\right) ; T_{F}: F \rightarrow H \text { with } D \subset F \subset M \text { metrically }\right\}
$$

where $T_{F}$ is a nonexpansive extension of $T$. We have $(T, D) \in \mathcal{C}$. Therefore, $\mathcal{C}$ is not empty. On the other hand, one can order partially $\mathcal{C}$ by $\left(T_{F}, F\right) \prec\left(T_{G}, G\right)$ if and only if $F \subset G$ and the restriction of $T_{G}$ to $F$ is $T_{F}$.

It is easy to see that $\mathcal{C}$ satisfies the hypothesis of Zorn's lemma. Therefore, $\mathcal{C}$ has maximal elements. Let $\left(T_{1}, F_{1}\right)$ be one maximal element of $\mathcal{C}$. Let us show that $F_{1}=M$. Assume not. Let $z \in M \backslash F_{1}$ and set $F=F_{1} \cup\{z\}$. Let us extend $T_{1}$ to $F$. The question is to find a point $z_{1}$, which will play the role of the value of the extension at $z$. Since we need the extension to be nonexpansive, we must have $d\left(T_{1}(x), z_{1}\right) \leq d(x, z)$ for all $x \in F_{1}$. Consider the family of closed balls $\left\{B\left(T_{1}(x), d(x, z)\right)\right\}$, with $x \in F_{1}$. Since $d\left(T_{1}(x), T_{1}(y)\right) \leq d(x, y) \leq d(x, z)+d(z, y)$ for all $x, y \in F_{1}$, the hyperconvexity of $H$ implies $\cap_{x \in F_{1}} B\left(T_{1}(x), d(x, z)\right) \neq \emptyset$. Let $z_{1}$ be any point in this intersection. Set $T^{*}: F \rightarrow H$ by

$$
T^{*}(x)= \begin{cases}T_{1}(x) & \text { if } x \neq z \\ z_{1} & \text { if } x=z .\end{cases}
$$

It is easy to check that $\left(T^{*}, F\right)$ belongs to $\mathcal{C}$, hence $\left(T_{1}, F_{1}\right) \prec\left(T^{*}, F\right)$ and $\left(T_{1}, F_{1}\right) \neq\left(T^{*}, F\right)$. This contradicts the maximality of $\left(T_{1}, F_{1}\right)$. Therefore, $F_{1}=M$. In other words, $T$ has a nonexpansive extension to $M$.

Conversely, assume that $H$ is injective, i.e. for every metric space $D$ and every nonexpansive map $T: D \rightarrow H$, there exists a nonexpansive extension $T^{*}: M \rightarrow H$ of $T$, where $M$ is any metric space which contains $D$ metrically. Let us prove that $H$ is hyperconvex. Aronszajn and Panitchpakdi's original proof is divided into two parts. First, they showed that $H$ is metrically convex. Then they showed that it is hyperconvex. Here, we will try to attempt a proof using Isbell's ideas. Indeed, given $\left\{x_{\alpha}\right\}_{\alpha \in \Gamma}$ in $H$ and positive numbers $\left\{r_{\alpha}\right\}_{\alpha \in \Gamma}$ such that $d\left(x_{\alpha}, x_{\beta}\right) \leq$ $r_{\alpha}+r_{\beta}$ for any $\alpha$ and $\beta$ in $\Gamma$ we want to show that $\bigcap_{\alpha \in \Gamma} B\left(x_{\alpha}, r_{\alpha}\right) \neq \emptyset$. Without any loss of generality, we may assume that $x_{\alpha} \neq x_{\beta}$ for any $\alpha \neq \beta$. Consider the set $\mathcal{F}$ of positive real valued functions $f$ defined on the set $D=\left\{x_{\alpha} ; \alpha \in \Gamma\right\}$ such that $d\left(x_{\alpha}, x_{\beta}\right) \leq f\left(x_{\alpha}\right)+f\left(x_{\beta}\right)$ for any $\alpha, \beta \in \Gamma$. Note that the function $r: D \rightarrow \mathbb{R}$ defined by $r\left(x_{\alpha}\right)=r_{\alpha}$ belongs to this set. $\mathcal{F}$ is partially ordered by the pointwise order on the real line. Obviously, any descending chain of elements of $\mathcal{F}$ has a lower bound. Hence, Zorn's lemma implies the existence of a minimal element $f \in \mathcal{F}$ smaller than $r$, i.e. $f\left(x_{\alpha}\right) \leq r\left(x_{\alpha}\right)$, for any $\alpha \in \Gamma$.

Now, using the minimality of $f$, we can prove that $f\left(x_{\alpha}\right) \leq d\left(x_{\alpha}, x_{\beta}\right)+f\left(x_{\beta}\right)$ for any $\alpha$ and $\beta$ in $\Gamma$. Indeed, assume this is not the case. Then there exists $\alpha_{0}$ and $\beta_{0}$ such that $d\left(x_{\alpha_{0}}, x_{\beta_{0}}\right)+$ $f\left(x_{\beta_{0}}\right)<f\left(x_{\alpha_{0}}\right)$. Set

$$
F\left(x_{\gamma}\right)= \begin{cases}f\left(x_{\gamma}\right) & \text { if } \gamma \neq \alpha_{0} \\ d\left(x_{\alpha_{0}}, x_{\beta_{0}}\right)+f\left(x_{\beta_{0}}\right) & \text { if } \gamma=\alpha_{0}\end{cases}
$$

$F$ satisfies that $F \leq f$ and $F \neq f$, contradicting the minimality of $f$. Let $\omega$ be a point not in the set $\mathcal{H}$. Consider the set $D^{*}=D \cup\{\omega\}$. The distance between the elements of $D$ is the one inherited from $H$. For the new point, set $d\left(\omega, x_{\alpha}\right)=f\left(x_{\alpha}\right)$. It is easy to check that $D^{*}$ is a metric space which contains $D$ metrically. Our assumption assures us of the existence of a nonexpansive extension $R$ of the identity map (defined from $D$ into $H$ ). It is clear that 


$$
\begin{aligned}
d\left(R\left(x_{\alpha}\right), R(\omega)\right)=d\left(x_{\alpha}, R(\omega)\right) \leq f\left(x_{\alpha}\right) & \leq r_{\alpha} \text { for any } \alpha \in \Gamma, \text { and so } \\
R(\omega) & \in \bigcap_{\alpha \in \Gamma} B\left(x_{\alpha}, r_{\alpha}\right) \bigcap H,
\end{aligned}
$$

which completes the proof.

Remark 4.3. Clearly this answers the Hahn-Banach extension problem for nonexpansive mappings. In fact, Aronszajn and Panitchpakdi obtained a more general result involving modulus of uniform continuity. The main ingredients behind the proof are still the same. Note that in the second part of the proof, we extended an identity map. The extension mapping is in fact a retract. Recall that a map $R: M \rightarrow N$ is said to be a retraction if $N \subset M$ and $R$ restricted to $N$ is the identity, i.e. $R(n)=n$, for any $n \in N$. It is easy to see that this is equivalent to $R \circ R=R$. The set $N$ is therefore called a retract of $M$.

A similar result to Aronszajn and Panitchpakdi's main theorem may be stated in terms of retractions as follows.

Theorem 4.4. Let $H$ be a metric space. The following statements are equivalent:

(i) $H$ is hyperconvex;

(ii) for every metric space $M$ which contains $H$ metrically, there exists a nonexpansive retraction $R: M \rightarrow H$

(iii) for any point $\omega$ not in $H$, there exists a nonexpansive retraction $R: H \cup\{\omega\} \rightarrow H$.

The proof is similar to the one given for the main theorem. The last statement needs some additional explanations. Indeed, the idea here is to consider the hyperconvex metric space $l_{\infty}(H)$ (in which $H$ embeds isometrically). In order to show that an intersection of balls in $H$ is not empty, embed the balls in $l_{\infty}(H)$. Then use the hyperconvexity of $l_{\infty}(H)$ to show that the intersection is not empty. Take a point in the intersection and the retraction sends this point into the intersection of the balls in $H$.

Remark 4.5. Note that statement (ii) is also known as an absolute retract property. This is why hyperconvex metric spaces are also called absolute nonexpansive retract (or in short ANR). The statement (iii) is quite surprising, since we do not extend to any bigger set but only to a point. So this behavior may be seen as a local behavior versus a global one for statement (ii).

Using the above remark, the second author [32] introduced the concept of 1-local retract of a metric space.

Definition 4.6. Let $M$ be a metric space. A subset $N$ is called a 1-local retract of $M$ if for any point $x \in M \backslash N$, there exists a nonexpansive retraction $R: M \cup\{x\} \rightarrow N$.

If we take in this definition the set $M$ to be any metric space which contains $N$ metrically, the 1-local retract property becomes absolute 1-local retract property. Note that absolute 1-local retracts are absolute nonexpansive retract, i.e. hyperconvex.

Remark 4.7. In the above definition, the number 1 stands for the nonexpansive behavior of the retract. In other words, one may define $\lambda$-local retract as well by assuming that the retraction is Lipschitzian with Lipschitz constant equal to $\lambda$. 
Notice that statement (ii), in the above theorem, has a nice extension. Indeed, we have the following result:

Corollary 4.8. Let $H$ be a metric space. The following statements are equivalent:

(i) $H$ is hyperconvex;

(ii) there exist a hyperconvex metric space $H^{*}$ which contains $H$ metrically and a nonexpansive retraction $R: H^{*} \rightarrow H$;

In other words, a nonexpansive retract of a hyperconvex metric space is also hyperconvex. The proof is elementary.

Remark 4.9. This result was used by the second author in [31] to prove that hyperconvex metric spaces enjoy a better convexity property than the one described previously. Indeed, let $H$ be a hyperconvex metric space. We have seen that $H$ embeds isometrically into $l_{\infty}(H)$. Corollary 4.8 implies the existence of a nonexpansive retraction $R: l_{\infty}(H) \rightarrow H$. Let $x_{1}, \ldots, x_{n}$ be in $H$ and positive numbers $\alpha_{1}, \ldots, \alpha_{n}$ such that $\sum_{i=1}^{i=n} \alpha_{i}=1$. In $l_{\infty}(H)$, consider the convex linear combination $\sum_{i=1}^{i=n} \alpha_{i} x_{i}$. Set $\bigoplus_{1 \leq i \leq n} \alpha_{i} x_{i}=R\left(\sum_{i=1}^{i=n} \alpha_{i} x_{i}\right) \in H$. Then, for any $\omega \in H$, we have

$$
d\left(\bigoplus_{1 \leq i \leq n} \alpha_{i} x_{i}, \omega\right)=d\left(R\left(\sum_{i=1}^{i=n} \alpha_{i} x_{i}\right), R(\omega)\right) \leq\left\|\sum_{i=1}^{i=n} \alpha_{i} x_{i}-\omega\right\|_{l_{\infty}(H)}
$$

which implies

$$
d\left(\bigoplus_{1 \leq i \leq n} \alpha_{i} x_{i}, \omega\right) \leq \sum_{i=1}^{i=n} \alpha_{i} d\left(x_{i}, \omega\right)
$$

We will rewrite the above inequality as

$$
d\left(\bigoplus_{1 \leq i \leq n} \alpha_{i} x_{i}, \cdot\right) \leq \sum_{i=1}^{i=n} \alpha_{i} d\left(x_{i}, \cdot\right)
$$

In fact we have a more general formula

$$
d\left(\bigoplus_{1 \leq i \leq n} \alpha_{i} x_{i}, \bigoplus_{1 \leq i \leq n} \alpha_{i} y_{i}\right) \leq \sum_{1 \leq i \leq n} \alpha_{i} d\left(x_{i}, y_{i}\right)
$$

One may argue that the choice of our convex combination in $H$ depends on the retraction $R$ and the choice of the isometric embedding, the answer is yes. Therefore depending on the problem, one may have to be careful about this choice.

Sine [55] began the study of the retraction property in hyperconvex spaces in a little more detailed way than what we have stated so far. His results are crucial in investigating nonexpansive mappings defined on hyperconvex metric spaces. We will begin the final part of this section focusing on admissible subsets, for which Sine proved the following relevant fact. For any positive real number $r$ and any set $A$ in a metric space $M$, we define the $r$-parallel set of $A$ as

$$
A+r=\bigcup\{B(a, r) ; a \in A\} .
$$

We have the following result. 
Lemma 4.10. Let $H$ be a hyperconvex metric space. Let $J$ be an admissible subset of $H$. Set $J=\cap_{\alpha \in \Gamma} B\left(x_{\alpha}, r_{\alpha}\right)$. Then for any $r \geq 0$, we have

$$
J+r=\bigcap_{\alpha \in \Gamma} B\left(x_{\alpha}, r_{\alpha}+r\right) .
$$

In other words, the r-parallel of an admissible subset of a hyperconvex metric space is also an admissible set (this is not a common property of metric spaces).

Proof. Let $y \in J+r$. Then there exists $a \in J$ such that $d(y, a) \leq r$. Hence $d\left(x_{\alpha}, y\right) \leq$ $\left.\overline{d\left(x_{\alpha}, a\right.}\right)+d\left(a, x_{\alpha}\right) \leq r_{\alpha}+r$ for all $\alpha \in \Gamma$. Thus

$$
J+r \subset \bigcap_{\alpha \in \Gamma} B\left(x_{\alpha}, r_{\alpha}+r\right)
$$

For the reverse inclusion, let $y \in \bigcap_{\alpha \in \Gamma} B\left(x_{\alpha}, r_{\alpha}+r\right)$. So we have $d\left(x_{\alpha}, y\right) \leq r_{\alpha}+r$ for all $\alpha \in \Gamma$. Now, since $H$ is hyperconvex, $\bigcap_{\alpha \in \Gamma} B\left(x_{\alpha}, r_{\alpha}\right) \cap B(y, r) \neq \emptyset$ which is equivalent to $J \cap B(y, r) \neq \emptyset$. This clearly implies that $y \in J+r$.

Remark 4.11. For any positive real number $r$ and any set $A$ in a metric space $M$, we define the r-neighborhood

$$
N_{r}(A)=\{x \in M ; \operatorname{dist}(x, A) \leq r\}
$$

If $A$ is proximinal, i.e. for every $x \in M$ there exists $a \in A$ such that $\operatorname{dist}(x, A)=d(x, a)$, then the r-parallel set $A+r$ and the $r$-neighborhood $N_{r}(A)$ are identical. This is the case for example if $A$ is externally hyperconvex in a hyperconvex metric space $M$.

Before we state Sine's result recall that a map $T$ defined on a metric space $M$ is said to be $\varepsilon$-constant if $d(x, T x) \leq \varepsilon$ for all $x \in M$. We have the following result:

Theorem 4.12. Let $H$ be a hyperconvex metric space. Let $J$ be a nonempty admissible subset of $H$. Then for any $\varepsilon>0$ there exists an $\varepsilon$-constant nonexpansive retraction of the parallel set $J_{\varepsilon}=J+\varepsilon$ onto $J$.

Proof. We have observed above that $J_{\varepsilon}$ is a nonempty ball intersection and so is itself a hyper-

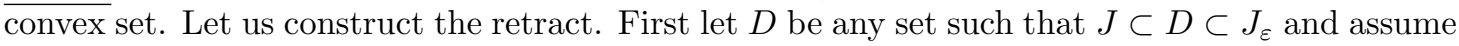
that $\Pi_{D}: D \rightarrow J$ is a $\varepsilon$-constant nonexpansive retract. Let $x \in J_{\varepsilon}-D$. Set $D^{*}=D \cup\{x\}$. Define the target set

$$
\operatorname{targ}(x)=\bigcap_{w \in D} B\left(\Pi_{D}(w), d(w, x)\right) \bigcap J \bigcap B(x, \varepsilon) .
$$

We claim that $\operatorname{targ}(x)$ is not empty. Indeed, set $J=\bigcap B\left(x_{\alpha}, r_{\alpha}\right)$. Then the set $\operatorname{targ}(x)$ is an intersection of balls. Since $H$ is hyperconvex, then to prove that $\operatorname{targ}(x)$ is not empty, it is enough to show that the balls intersect each other. Since $x \in J_{\varepsilon}$, then $B(x, \varepsilon)$ intersect any ball $B\left(x_{\alpha}, r_{\alpha}\right)$ (coming from $J)$. On the other hand, we have $d\left(\Pi_{D}(w), x\right) \leq d\left(\Pi_{D}(w), w\right)+d(w, x) \leq \varepsilon+d(x, w)$ because $\Pi_{D}$ is an $\varepsilon$-constant map. This clearly implies $B(x, \varepsilon) \cap B\left(\Pi_{D}(w), d(w, x)\right) \neq \emptyset$, for any $w \in D$. Since $\Pi_{D}$ maps into $J$, then $B\left(x_{\alpha}, r_{\alpha}\right) \cap B\left(\Pi_{D}(w), d(w, x)\right) \neq \emptyset$, for any $\alpha$. Therefore $\operatorname{targ}(x)$ is not empty. Let $z \in \operatorname{targ}(x)$ and define $\Pi^{*}: D^{*} \rightarrow J$ by

$$
\Pi^{*}(v)=\left\{\begin{array}{cl}
\Pi_{D}(v) & \text { if } v \in D \\
z & \text { if } v=x
\end{array}\right.
$$


It is easy to check that $\Pi^{*}$ is a $\varepsilon$-constant nonexpansive extension of $\Pi_{D}$. The rest of the proof is a simple induction. Start with $D=J$ and $\Pi_{D}$ to be the identity map on $D$. Zorn's lemma assures us of the existence of a maximal element $\left(D, \Pi_{D}\right)$. The argument described above will force the set $D$ to be $J_{\varepsilon}$, which completes the proof.

Remark 4.13. Now one may ask whether the previous result is also true for weakly externally hyperconvex or externally hyperconvex subsets. The answer is yes. In fact the same proof we did for admissible subsets still works for externally hyperconvex subsets without major modifications. But something more precise may be said about this problem since weak externally hyperconvexity actually characterizes those sets for which the conclusion of the previous theorem holds. We do not plan to show the proof of this result in this text which may be found in [15], however the following theorem is a partial result in that sense, in fact it states a reciprocal of Theorem 4.12.

Theorem 4.14. If $E$ is a subset of a hyperconvex metric space $H$ with the property that for each $z \in H \backslash E$ there exists a nonexpansive retraction $R: E \cup\{z\} \rightarrow E$ such that $d(z, R(z))=\operatorname{dist}(z, E)$, then $E$ is weakly externally hyperconvex.

Proof. The first we notice is that, from Theorem 4.4, such an $E$ must be a hyperconvex subset of $H$, i.e. $E$ is hyperconvex itself. We are going to assume that $E$ is not weakly externally hyperconvex in order to find $z \in H$ such that

$$
B(z, \operatorname{dist}(z, E)) \cap\left(\cap_{v \in D} B(v, d(v, z))\right) \cap E=\emptyset .
$$

This clearly contradicts the hypothesis of the theorem. We state (claim 1 ) that if $E$ is not weakly externally hyperconvex then there exist $z \in H$, a family $\left\{v_{\alpha}: \alpha \in A\right\}$ in $E$, and a family $\left\{r_{\alpha}\right\}$ in $\mathbb{R}^{+}$for which $d\left(v_{\alpha}, v_{\beta}\right) \leq r_{\alpha}+r_{\beta}, d\left(v_{\alpha}, z\right) \leq r_{\alpha}+\operatorname{dist}(z, E)$, and such that

$$
B(z, \operatorname{dist}(z, E)) \cap\left(\cap_{\alpha} B\left(v_{\alpha}, r_{\alpha}\right)\right) \cap E=\emptyset .
$$

However, since $M$ is hyperconvex it must be the case that

$$
D_{1}=B(z, \operatorname{dist}(z, E)) \cap\left(\cap_{\alpha} B\left(v_{\alpha}, r_{\alpha}\right)\right) \neq \emptyset .
$$

If $B(z$, dist $(z, E)) \cap\left(\cap_{v \in E} B(v, d(v, z))\right) \cap E=\emptyset$ we are done. Otherwise we proceed as follows. Since $D_{1} \cap E=\emptyset$ there is no loss of generality (claim 2) assuming that,

$$
\operatorname{dist}\left(B(z, \operatorname{dist}(z, E)) \cap E, D_{1}\right)=d>0 .
$$

It is possible to choose $w_{1} \in D_{1}$ and $w \in B(z$, dist $(z, E)) \cap E$ so that $d\left(w_{1}, w\right)=d+\varepsilon$ for sufficiently small $\varepsilon>0$. Now, by hyperconvexity of $M$,

$$
B\left(z, \operatorname{dist}(z, E)-\frac{d+\varepsilon}{2}\right) \cap B\left(w_{1}, \frac{d+\varepsilon}{2}\right) \cap B\left(w, \frac{d+\varepsilon}{2}\right) \neq \emptyset .
$$

Let $z_{1}$ be any point in this intersection. Notice that if $d\left(z_{1}, p\right)<\frac{d+\varepsilon}{2}$ for any $p \in E$, then

$$
\begin{aligned}
d(z, p) & \leq d\left(z, z_{1}\right)+d\left(z_{1}, p\right) \\
& <\operatorname{dist}(z, E)-\frac{d+\varepsilon}{2}+\frac{d+\varepsilon}{2} \\
& =\operatorname{dist}(z, E)
\end{aligned}
$$

which is a contradiction. Since $\operatorname{dist}\left(z_{1}, E\right) \leq d\left(z_{1}, w\right) \leq \frac{d+\varepsilon}{2}$, we conclude $\operatorname{dist}\left(z_{1}, E\right)=\frac{d+\varepsilon}{2}$.

By the assumptions of the theorem, the set

$$
B\left(z_{1}, \operatorname{dist}\left(z_{1}, E\right)\right) \cap\left(\cap_{\alpha} B\left(v_{\alpha}, d\left(v_{\alpha}, z_{1}\right)\right)\right) \cap E
$$


would have to be nonempty. However, since $d\left(z_{1}, v_{\alpha}\right) \leq r_{\alpha}+\frac{d+\varepsilon}{2}$, for each $\alpha$, we have

$$
\begin{aligned}
& B\left(z_{1}, \operatorname{dist}\left(z_{1}, E\right)\right) \cap\left(\cap_{\alpha} B\left(v_{\alpha}, d\left(v_{\alpha}, z_{1}\right)\right)\right) \\
& \subseteq B(z, \operatorname{dist}(z, E)) \cap\left(\cap_{\alpha} B\left(v_{\alpha}, r_{\alpha}+\frac{d+\varepsilon}{2}\right)\right),
\end{aligned}
$$

and by Lemma 4.10 ,

$$
B(z, \operatorname{dist}(z, E)) \cap\left(\cap_{\alpha} B\left(v_{\alpha}, r_{\alpha}+\frac{d+\varepsilon}{2}\right)\right) \subseteq N_{\frac{d+\varepsilon}{2}}\left(D_{1}\right) .
$$

Clearly this neighborhood of $D_{1}$ cannot intersect $E \cap B(z, \operatorname{dist}(z, E))$ for $\varepsilon>0$ sufficiently small, so we conclude

$$
B\left(z_{1}, \operatorname{dist}\left(z_{1}, E\right)\right) \cap\left(\cap_{\alpha} B\left(v_{\alpha}, d\left(v_{\alpha}, z_{1}\right)\right)\right) \cap E=\emptyset .
$$

Proof of Claim 1. Notice that what we directly get from the negation of the definition of weak externally hyperconvexity is that there exist $z \in M$, a family $\left\{v_{\alpha}: \alpha \in A\right\}$ in $E$, and a family $\left\{r_{\alpha}\right\} \cup\left\{r_{z}\right\}$ in $\mathbb{R}$ such that $r_{z} \geq \operatorname{dist}(z, E), d\left(v_{\alpha}, v_{\beta}\right) \leq r_{\alpha}+r_{\beta}, d\left(v_{\alpha}, z\right) \leq r_{\alpha}+r_{z}$, and for which

$$
B(z, \operatorname{dist}(z, E)) \cap\left(\cap_{\alpha} B\left(v_{\alpha}, r_{\alpha}\right)\right) \cap E=\emptyset,
$$

something slightly different to which is assured by our claim. In order to prove the claim, set $r_{\alpha}^{\prime}=r_{\alpha}+r_{z}-\operatorname{dist}(z, E)$, and $r_{z}^{\prime}=\operatorname{dist}(z, E)$ then we state that the intersection

$$
B(z, \operatorname{dist}(z, E)) \cap\left(\cap_{\alpha} B\left(v_{\alpha}, r_{\alpha}^{\prime}\right)\right) \cap E=\emptyset .
$$

Otherwise fix $u$ in this intersection and consider $\cap_{\alpha} B\left(v_{\alpha}, r_{\alpha}\right) \cap B\left(u, r_{z}-\operatorname{dist}(z, E)\right)$. From the hyperconvexity of $E$, this intersection would also be nonempty but, since this set is contained in the set intersection of (1), this would be a contradiction.

Proof of Claim 2. To prove this second claim, assume $d=0$ and let $\left\{\varepsilon_{n}\right\}$ be a decreasing sequence of positive numbers null-convergent. Select $u_{1} \in B(z, \operatorname{dist}(z, E)) \cap E \cap\left(\cap_{\alpha} B\left(v_{\alpha}, r_{\alpha}+\varepsilon_{1}\right)\right)$, and consider $B(z, \operatorname{dist}(z, E)) \cap E \cap B\left(u_{1}, \varepsilon_{1}+\varepsilon_{2}\right) \cap\left(\cap_{\alpha} B\left(v_{\alpha}, r_{\alpha}+\varepsilon_{2}\right)\right)$. If this intersection is empty add $B\left(u_{1}, \varepsilon_{1}\right)$ to the family $\left\{B\left(v_{\alpha}, r_{\alpha}\right)\right\}$ and so we have a new family for which $d$ is positive. Otherwise, select $u_{2}$ in this in this intersection and consider $B(z, \operatorname{dist}(z, E)) \cap E \cap B\left(u_{2}, \varepsilon_{2}+\right.$ $\left.\varepsilon_{3}\right) \cap\left(\cap_{\alpha} B\left(v_{\alpha}, r_{\alpha}+\varepsilon_{3}\right)\right)$, and repeat the previous step. Either this process terminates after a finite number of steps, providing a new family for which $d>0$, or we obtain a Cauchy sequence $\left\{u_{n}\right\}$ whose limit lies in $D_{1} \cap E$, which is a contradiction.

We finish this section with the announced-characterizing result which follows from Theorems 4.14 and 9.12 .

Theorem 4.15. Let $H$ be a hyperconvex metric space. Then $E \subset H$ is weakly externally hyperconvex if and only if for any $\varepsilon>0$ there exists an $\varepsilon$-constant nonexpansive retract of the parallel set $E+\varepsilon$ onto $E$.

What else do we know about hyperconvex sets? May be one of the most elegant results in hyperconvex spaces is Baillon's theorem. We will discuss this and others in the next section. 


\section{More on HyperConveX SPACES}

Hyperconvexity, as we mentioned before, is an intersection property. In other words, if a metric space is hyperconvex then the family of admissible sets has some kind of compactness behavior. Indeed, let $M$ be a metric space and consider the family of admissible sets $\mathcal{A}(M)$. Following Penot's definition, we will say that $\mathcal{A}(M)$ is compact if for any family $\left\{A_{\alpha}\right\}$ of elements in $\mathcal{A}(M)$, we have

$$
\bigcap_{\alpha} A_{\alpha} \neq \emptyset
$$

provided finite intersections are not empty. Hyperconvexity obviously implies that $\mathcal{A}(M)$ is compact. But this kind of compactness is not equivalent to hyperconvexity in general. Indeed, hyperconvexity is about balls intersecting each others. For example, Lindenstrauss [45] showed that balls of $l_{1}$ have a very nice and similar intersection property though $l_{1}$ is not hyperconvex. Indeed, he showed that any collection of balls has a nonempty intersection provided the balls intersect three by three. In fact, in any reflexive Banach space or dual space $X$, the family $\mathcal{A}(X)$ is compact under Penot's formulation. Recall that $l_{\infty}(I)$ is basically the only hyperconvex Banach space. The reader will find more about the relation between hyperconvexity and Banach spaces in $[19,29,42,45,46]$.

Early investigators of hyperconvexity wondered whether the compactness of the family of admissible sets holds also for the family of hyperconvex sets. Notice that this family is not stable under intersection. Indeed, the intersection of two hyperconvex sets may not be hyperconvex, even in the plane. So it was asked whether any descending chain of nonempty hyperconvex sets has a nonempty intersection. This question was answered by Baillon [3] in a highly technical proof. It is not known to us if a simple proof of this result exists.

Theorem 5.1. Let $M$ be a bounded metric space. Let $\left(H_{\beta}\right)_{\beta \in \Gamma}$ be a decreasing family of nonempty hyperconvex subsets of $M$, where $\Gamma$ is totally ordered. Then $\bigcap_{\beta \in \Gamma} H_{\beta}$ is not empty and is hyperconvex.

Proof. Consider the family

$$
\mathcal{F}=\left\{A=\prod_{\beta \in \Gamma} A_{\beta} ; A_{\beta} \in \mathcal{A}\left(H_{\beta}\right) \text { and }\left(A_{\beta}\right) \text { is decreasing and nonempty }\right\} .
$$

Since $M$ is bounded, then each $H_{\beta}$ is bounded and therefore we must have $H_{\beta} \in \mathcal{A}\left(H_{\beta}\right)$. So $\mathcal{F}$ is not empty since $\prod_{\beta \in \Gamma} H_{\beta} \in \mathcal{F}$. Since $H_{\beta}$ is hyperconvex, $\mathcal{A}\left(H_{\beta}\right)$ is compact for every $\beta \in \Gamma$. Therefore, $\mathcal{F}$ satisfies the assumptions of Zorn's lemma when ordered by set inclusion. Hence for every $D \in \mathcal{F}$ there exists a minimal element $A \in \mathcal{F}$ such that $A \subset D$. We claim that if $A=\prod_{\beta \in \Gamma} A_{\beta}$ is minimal then there exists $\beta_{0} \in \Gamma$ such that $\delta\left(A_{\beta}\right)=0$ for every $\beta \geq \beta_{0}$. Indeed, let $\beta \in \Gamma$ be fixed. For every $D \subset M$, set

$$
\operatorname{cov}_{\beta}(D)=\bigcap_{x \in H_{\beta}} B\left(x, r_{x}(D)\right) .
$$

Consider $A^{\prime}=\prod_{\alpha \in \Gamma} A_{\alpha}^{\prime}$ where

$$
\begin{cases}A_{\alpha}^{\prime}=\operatorname{cov}_{\beta}\left(A_{\beta}\right) \cap A_{\alpha} & \text { if } \alpha \leq \beta \\ A_{\alpha}^{\prime}=A_{\alpha} & \text { if } \alpha \geq \beta .\end{cases}
$$

The family $\left(A_{\alpha \geq \beta}^{\prime}\right)$ is decreasing since $A \in \mathcal{F}$. Let $\alpha \leq \gamma \leq \beta$. Then $A_{\gamma}^{\prime} \subset A_{\alpha}^{\prime}$ since $A_{\gamma} \subset A_{\alpha}$ and $A_{\beta}=\operatorname{cov}_{\beta}\left(A_{\beta}\right) \cap A_{\beta}$. Hence the family $\left(A_{\alpha}^{\prime}\right)$ is decreasing. On the other hand if $\alpha \leq \beta$, 
then $\operatorname{cov}_{\beta}\left(A_{\beta}\right) \cap A_{\alpha} \in \mathcal{A}\left(H_{\alpha}\right)$ since $H_{\beta} \subset H_{\alpha}$. Hence $A_{\alpha}^{\prime} \in \mathcal{A}\left(H_{\alpha}\right)$. Therefore, we have $A^{\prime} \in \mathcal{F}$. Since $A$ is minimal, $A=A^{\prime}$ which implies

$$
A_{\alpha}=\operatorname{cov}_{\beta}\left(A_{\beta}\right) \cap A_{\alpha} \text {, for every } \alpha \leq \beta .
$$

Let $x \in H_{\beta}$ and $\alpha \leq \beta$. Since $A_{\beta} \subset A_{\alpha}$, then $r_{x}\left(A_{\beta}\right) \leq r_{x}\left(A_{\alpha}\right)$. Because $\operatorname{cov}_{\beta}\left(A_{\beta}\right)=$ $\bigcap_{x \in H_{\beta}} B\left(x, r_{x}\left(A_{\beta}\right)\right)$, then we have $\operatorname{cov}_{\beta}\left(A_{\beta}\right) \subset B\left(x, r_{x}\left(A_{\beta}\right)\right)$ which implies $r_{x}\left(\operatorname{cov}_{\beta}\left(A_{\beta}\right)\right) \leq r_{x}\left(A_{\beta}\right)$. Additionally $A_{\alpha} \subset \operatorname{cov}_{\beta}\left(A_{\beta}\right)$ so $r_{x}\left(A_{\beta}\right) \leq r_{x}\left(A_{\alpha}\right) \leq r_{x}\left(\operatorname{cov}_{\beta}\left(A_{\beta}\right)\right) \leq r_{x}\left(A_{\beta}\right)$. Therefore, we have $r_{x}\left(A_{\alpha}\right)=r_{x}\left(A_{\beta}\right)$ for every $x \in H_{\beta}$. Using the definition of $r$, we get $r\left(A_{\alpha}\right) \leq r\left(A_{\beta}\right)$. Let $a \in A_{\alpha}$ and set $s=r_{a}\left(A_{\alpha}\right)$. Then $a \in \operatorname{cov}_{\beta}\left(A_{\beta}\right)$ since $A_{\alpha} \subset \operatorname{cov}_{\beta}\left(A_{\beta}\right)$. Hence $a \in$ $\bigcap_{x \in A_{\beta}} B(x, s) \cap \operatorname{cov}_{\beta}\left(A_{\beta}\right)$. So, from the hyperconvexity of $H_{\beta}$,

$$
S_{\beta}=H_{\beta} \bigcap \bigcap_{x \in A_{\beta}} B(x, s) \bigcap \operatorname{cov}_{\beta}\left(A_{\beta}\right) \neq \emptyset .
$$

Let $z \in S_{\beta}$, then $z \in \bigcap_{x \in A_{\beta}} B(x, s)$ and, since $A_{\beta}=H_{\beta} \bigcap \operatorname{cov}_{\beta}\left(A_{\beta}\right)$, it follows that $r_{z}\left(A_{\beta}\right) \leq s$, which implies $r\left(A_{\beta}\right) \leq s=r_{a}\left(A_{\alpha}\right)$ for every $a \in A_{\alpha}$. Hence $r\left(A_{\beta}\right) \leq r\left(A_{\alpha}\right)$. Therefore we have $r\left(A_{\beta}\right)=r\left(A_{\alpha}\right)$, for every $\alpha, \beta \in \Gamma$.

Assume that $\delta\left(A_{\beta}\right)>0$ for every $\beta \in \Gamma$. Set $A_{\beta}^{\prime \prime}=C\left(A_{\beta}\right)$ for every $\beta \in \Gamma$. The family $\left(A_{\beta}^{\prime \prime}\right)$ is decreasing. Indeed, let $\alpha \leq \beta$ and $x \in A_{\beta}^{\prime \prime}$. Then we have $r_{x}\left(A_{\beta}\right)=r\left(A_{\beta}\right)$. Since we proved that $r_{z}\left(A_{\beta}\right)=r_{z}\left(A_{\alpha}\right)$ for every $z \in H_{\beta}$, then $r_{x}\left(A_{\alpha}\right)=r_{x}\left(A_{\beta}\right)=r\left(A_{\beta}\right)=r\left(A_{\alpha}\right)$, which implies that $x \in A_{\alpha}^{\prime \prime}$. Therefore, we have $A^{\prime \prime}=\prod_{\beta \in \Gamma} A_{\beta}^{\prime \prime} \in \mathcal{F}$. Since $A^{\prime \prime} \subset A$ and $A$ is minimal, we get $A=A^{\prime \prime}$. Therefore, we have $C\left(A_{\beta}\right)=A_{\beta}$ for every $\beta \in \Gamma$. This contradicts the fact that $H_{\beta}$ is hyperconvex for every $\beta \in \Gamma$. Hence there exists $\beta_{0} \in \Gamma$ such that $\delta\left(A_{\beta}\right)=0$, for every $\beta \geq \beta_{0}$. The proof of our claim is therefore complete since we have $A_{\beta}=\{a\}$ for every $\beta \geq \beta_{0}$ which clearly implies that $a \in \bigcap_{\beta \in \Gamma} H_{\beta} \neq \emptyset$.

In order to complete the proof, we need to show that $S=\bigcap_{\beta \in \Gamma} H_{\beta}$ is hyperconvex. Let $\left(B_{i}\right)_{i \in I}$ be a family of balls centered in $S$ such that $\bigcap_{i \in I} B_{i} \neq \emptyset$. Set $D_{\beta}=\bigcap_{i \in I} B_{i} \bigcap H_{\beta}$ for $\beta \in \Gamma$. Since $H_{\beta}$ is hyperconvex and the family $\left(B_{i}\right)$ is centered in $H_{\beta}$, then $D_{\beta}$ is not empty and $D_{\beta} \in \mathcal{A}\left(H_{\beta}\right)$. Therefore, $D_{\beta}$ is hyperconvex. The above proof shows that $\bigcap_{\beta \in \Gamma} D_{\beta} \neq \emptyset$ which completes the proof of Theorem 5.1.

Remark 5.2. This proof is different from Baillon's original one. It is little more complicated. But it has the advantage of being easy to adapt to 1-local retract. In other words, the conclusion of Baillon's result holds for 1-local retract sets.

While the intersection of two admissible subsets of a given hyperconvex space is again admissible, in general it is not the case that the intersection of two hyperconvex subspaces of a hyperconvex space is itself hyperconvex, even if one of them is admissible. However the following is true.

Lemma 5.3. Let $H$ be a hyperconvex metric space. Suppose $E \subset H$ is externally hyperconvex relative to $H$ and suppose $A$ is an admissible subset of $H$. Then $E \cap A$ is externally hyperconvex relative to $H$.

Proof. Suppose $\left\{x_{\alpha}\right\}$ and $\left\{r_{\alpha}\right\}$ satisfy $d\left(x_{\alpha}, x_{\beta}\right) \leq r_{\alpha}+r_{\beta}$ and $\operatorname{dist}\left(x_{\alpha}, E \cap A\right) \leq r_{\alpha}$. Since $A$ is admissible, $A=\bigcap_{i \in I} B\left(x_{i} ; r_{i}\right)$ and $\operatorname{since} \operatorname{dist}\left(x_{\alpha}, E \cap A\right) \neq \emptyset$ it follows that $d\left(x_{\alpha}, x_{i}\right) \leq r_{\alpha}+r_{i}$ for 
each $i \in I$. Also, since $A \subset B\left(x_{i} ; r_{i}\right)$, it follows that $\operatorname{dist}\left(x_{i}, E \cap A\right) \leq r_{i}$ and that $d\left(x_{i}, x_{j}\right) \leq r_{i}+r_{j}$ for each $i, j \in I$. Therefore by external hyperconvexity of $E$

$$
\left(\bigcap_{i} B\left(x_{i} ; r_{i}\right)\right)\left(\bigcap_{\alpha} B\left(x_{\alpha}, r_{\alpha}\right)\right) \cap E=\bigcap_{\alpha} B\left(x_{\alpha}, r_{\alpha}\right) \cap(A \cap E) \neq \emptyset .
$$

This leads to the following.

Theorem 5.4. Let $\left\{H_{i}\right\}$ be a descending chain of nonempty externally hyperconvex subsets of a bounded hyperconvex space $H$. Then $\bigcap_{i} H_{i}$ is nonempty and externally hyperconvex in $H$.

Proof. Theorem 5.1 assures that $D=\bigcap_{i} H_{i} \neq \emptyset$. To see that $D$ is externally hyperconvex let $\left\{x_{\alpha}\right\} \subset H$ and $\left\{r_{\alpha}\right\} \subset \mathbb{R}$ satisfy $d\left(x_{\alpha}, x_{\beta}\right)^{i} \leq r_{\alpha}+r_{\beta}$ and $\operatorname{dist}\left(x_{\alpha}, D\right) \leq r_{\alpha}$. Since $H$ is hyperconvex we know that $A=\bigcap_{\alpha} B\left(x_{\alpha} ; r_{\alpha}\right) \neq \emptyset$. Also, since $\operatorname{dist}\left(x_{\alpha}, D\right) \leq r_{\alpha}$ we have $\operatorname{dist}\left(x_{\alpha}, H_{i}\right) \leq r_{\alpha}$ for each $i$, so, by external hyperconvexity of $H_{i}$, we conclude $A \cap H_{i} \neq \emptyset$ for each $i$. By Lemma 5.3 $\left\{A \cap H_{i}\right\}$ is a descending chain of nonempty hyperconvex subsets of $H$, so again by Theorem 5.1 $\bigcap_{i}\left(A \cap H_{i}\right)=A \cap D \neq \emptyset$.

Remark 5.5. Whether Lemma 5.3 holds for weakly externally hyperconvex subsets is not known yet, however it is a non very complicated exercise to prove that Theorem 5.4 is true for these subsets. An interesting open question is whether the intersection of two weakly externally hyperconvex subsets is still weakly externally hyperconvex. Such a property is a very important one from a structural point of view and it should help to show new fixed point results for weakly externally hyperconvex subsets that are already known for admissible subsets.

One of the implications of Theorem 5.1 is the existence of hyperconvex closures. Indeed, let $M$ be a metric space and consider the family $\mathcal{H}(M)=\{H ; H$ is hyperconvex and $M \subset H\}$. In view of what we said previously, the family $\mathcal{H}(M)$ is not empty. Using Baillon's result, any descending chain of elements of $\mathcal{H}(M)$ has a nonempty intersection. Therefore one may use Zorn's lemma which will insure us of the existence of minimal elements. These minimal hyperconvex sets are called hyperconvex hulls. Isbell was among the first to investigate the properties of the hyperconvex hulls. In fact he was the first one to give a concrete construction of a hyperconvex hull in [24]. We will discuss his ideas in Section 8.

It is clear that hyperconvex hulls are not unique. But they do enjoy some kind of uniqueness. Indeed, we have:

Proposition 5.6. Let $M$ be a metric space. Assume that $H_{1}$ and $H_{2}$ are two hyperconvex hulls of $M$. Then $H_{1}$ and $H_{2}$ are isometric.

Proof. Since $H_{1}$ and $H_{2}$ are hyperconvex, then they are injective. So there exists a nonexpansive map $T_{1}: H_{1} \rightarrow H_{2}$ such that the restriction of $T_{1}$ to $M$ is the identity map. Keep in mind that $H_{1}$ as well as $H_{2}$ contains $M$ isometrically. For the same reason, there exists also another nonexpansive map $T_{2}: H_{2} \rightarrow H_{1}$ such that the restriction of $T_{2}$ to $M$ is the identity map. We claim that $T_{1} \circ T_{2}$ is the identity map of $H_{2}$. Indeed, the map $T_{1} \circ T_{2}$ is defined on $H_{2}$ into $H_{2}$. Its restriction to $M$ is the identity map of $M$. So we have $M \subset \operatorname{Fix}\left(T_{1} \circ T_{2}\right)$, where

$$
\operatorname{Fix}\left(T_{1} \circ T_{2}\right)=\left\{x \in H_{1} ; T_{1} \circ T_{2}(x)=x\right\} .
$$


In the next section, we will show that if $T$ is nonexpansive, then $\operatorname{Fix}(T)$ is hyperconvex (see Theorem 6.1), so, Since $T_{1} \circ T_{2}$ is nonexpansive, $\operatorname{Fix}\left(T_{1} \circ T_{2}\right)$ is hyperconvex and contains $M$. The minimality of $H_{2}$ implies

$$
\operatorname{Fix}\left(T_{1} \circ T_{2}\right)=H_{2},
$$

which completes the proof of our claim. A similar argument will show that $T_{2} \circ T_{1}$ is the identity map of $H_{1}$. So $T_{1}$ and $T_{2}$ are inverse from each other and are nonexpansive. Therefore both are isometric maps.

Remark 5.7. Though hyperconvex hulls are not unique, the previous proposition shows that up to an isometry they are indeed unique. It is quite an amazing result. From now on, we will denote the hyperconvex hull of $M$ by $h(M)$. Recall that if $M$ is a subset of a hyperconvex set $H$, then there exists a hyperconvex hull $h(M)$ such that $M \subset h(M) \subset H$.

Isbell, in his study of the hyperconvex hulls, showed that if $M$ is compact then $h(M)$ is also compact. As a generalization of this result, we discuss next some ideas developed by the first author and Lpez in $[14,16]$. Since their work involves measure of noncompactness, let us first give some definitions. For a wide treatment of these definitions the reader is refereed to [2].

Definition 5.8. Let $M$ be a metric space and let $\mathcal{B}(M)$ denote the collection of nonempty, bounded subsets of $M$. Then:

(i) The Kuratowski measure of noncompactness $\alpha: \mathcal{B}(M) \rightarrow[0, \infty)$ is defined by

$$
\alpha(A)=\inf \left\{\varepsilon>0 ; A \subset \bigcup_{i=1}^{i=n} A_{i} \text { with } A_{i} \in \mathcal{B}(M) \text { and } \operatorname{diam}\left(A_{i}\right) \leq \varepsilon\right\} .
$$

(ii) The Hausdorff (or ball) measure of noncompactness $\chi: \mathcal{B}(M) \rightarrow[0, \infty)$ is defined by

$$
\chi(A)=\inf \left\{r>0 ; A \subset \bigcup_{i=1}^{i=n} B\left(x_{i}, r\right) \text { with } x_{i} \in M\right\} .
$$

These two measures are very much related to each other and to compactness. Indeed, the following classical properties are well known.

(1) For any $A \in \mathcal{B}(M)$, we have $0 \leq \alpha(A) \leq \delta(A)=\operatorname{diam}(A)$.

(2) For any $A \in \mathcal{B}(M)$, we have $\alpha(A)=0$ if and only if $A$ is precompact.

(3) For any $A \in \mathcal{B}(M)$ and $B \in \mathcal{B}(M)$, we have $\alpha(A \cup B)=\max \{\alpha(A), \alpha(B)\}$.

(4) For any $A \in \mathcal{B}(M)$, we have $\chi(A) \leq \alpha(A) \leq 2 \chi(A)$.

(5) If $\left(A_{i}\right)_{i \in I}$ is a decreasing chain of closed bounded sets such that $\inf _{i \in I} \alpha\left(A_{i}\right)=0$, then $\bigcap_{i \in I} A_{i}$ is not empty and is compact, i.e. $\alpha\left(\bigcap_{i \in I} A_{i}\right)=0$.

In hyperconvex metric spaces, the two measures behave nicely. Indeed, we have:

Proposition 5.9. Let $H$ be a hyperconvex metric space and $A$ be a bounded subset of $H$. Then we have $\alpha(A)=2 \chi(A)$.

Proof. From (4) it is enough to prove that $2 \chi(A) \leq \alpha(A)$. Let $\varepsilon>\chi(A)$. Then there exist $\overline{A_{1}, \ldots,} A_{n}$ subsets of $A$ such that $A=\bigcup_{1 \leq i \leq n} A_{i}$ with $\operatorname{diam}\left(A_{i}\right) \leq \varepsilon$, for $i=1, \ldots, n$. From the hyperconvexity of $H$ for any $i \in\{1, \ldots, n\}$, there exists $h_{i} \in H$ such that $A_{i} \subset B\left(h_{i}, \frac{\varepsilon}{2}\right)$. Hence 
$A \subset \bigcup_{1 \leq i \leq n} B\left(h_{i}, \frac{\varepsilon}{2}\right)$, which gives $\alpha(A) \leq \frac{\varepsilon}{2}$. Hence $\alpha(A) \leq \frac{\chi(A)}{2}$ which completes the proof of our claim.

The following technical result will be needed later on. Note that this result may be seen as an adaptation of the classical Arzel -Ascoli Theorem.

Lemma 5.10. Let $M$ be a metric space. Consider the space $\lambda_{[a, b]}(M)$ of Lipschitzian real-valued functions defined on $M$ with Lipschitz constant less than $\lambda$ with values in the interval $[a, b]$. Then we have

$$
\alpha\left(\lambda_{[a, b]}(M)\right) \leq 2 \lambda \chi(M) .
$$

Proof. Let $\varepsilon_{0}>\chi(M)$. Without loss of generality, we may assume that there exist $x_{1}, \ldots, x_{n}$ in $M$ such that for any $x \in M$, there exists $i \in\{1, \ldots, n\}$ such that $d\left(x, x_{i}\right) \leq \varepsilon_{0}$. Since $[a, b]$ is compact, for any $\varepsilon>0$, there exist $c_{1}, \ldots, c_{m}$ in $[a, b]$ such that for any $c \in[a, b]$ there exists $i \in\{1, \ldots, m\}$ such that $\left|c-c_{i}\right| \leq \varepsilon$. Let $\psi:\{1, \ldots, n\} \rightarrow\{1, \ldots, m\}$ be an application. Define

$$
\lambda_{\psi}=\left\{f \in \lambda_{[a, b]}(M) ; \sup _{1 \leq i \leq n}\left|f\left(x_{i}\right)-c_{\psi(i)}\right| \leq \varepsilon\right\} .
$$

Though these sets may be eventually empty, we still have that

$$
\lambda_{[a, b]}(M)=\bigcup_{\psi \in\{1, \ldots, m\}\{1, \ldots, n\}} \lambda_{\psi} .
$$

Let $f, g \in \lambda_{\psi}$. For any $x \in M$, there exists $i \in\{1, \ldots, n\}$ such that $d\left(x, x_{i}\right) \leq \varepsilon_{0}$. Then we have

$$
|f(x)-g(x)| \leq\left|f(x)-f\left(x_{i}\right)\right|+\left|f\left(x_{i}\right)-g\left(x_{i}\right)\right|+\left|g\left(x_{i}\right)-g(x)\right|,
$$

which implies $|f(x)-g(x)| \leq \lambda \varepsilon_{0}+2 \varepsilon+\lambda \varepsilon_{0}$. Hence $\sup _{x \in M}|f(x)-g(x)| \leq 2 \lambda \varepsilon_{0}+2 \varepsilon$. Since the set $\{1, \ldots, m\}^{\{1, \ldots, n\}}$ is finite, we get $\alpha\left(\lambda_{[a, b]}(M)\right) \leq 2 \lambda \varepsilon_{0}+2 \varepsilon$. So, from the arbitrariness of $\varepsilon$, we get $\alpha\left(\lambda_{[a, b]}(M)\right) \leq 2 \lambda \varepsilon_{0}$, which clearly implies $\alpha\left(\lambda_{[a, b]}(M)\right) \leq 2 \lambda \chi(M)$.

We will show in Section 8 how Isbell constructed for any bounded metric space $M$ a hyperconvex hull $h(M)$ included in $\lambda_{[0, \delta]}(M)$, where $\lambda=1$ and $\delta$ is the diameter of $M$. So from the above lemma, we deduce the following result.

Corollary 5.11. Let $M$ be any bounded metric space and $h(M)$ its hyperconvex hull. Then we have $\chi(h(M))=\chi(M)$ and $\alpha(h(M))=\alpha(M)$.

Proof. Since $h(M)$ may be chosen such that $h(M) \subset \lambda_{[0, \delta]}(M)$, where $\lambda=1$ and $\delta$ is the diameter of $M$, it follows $\alpha(h(M)) \leq 2 \chi(M)$. Using the previous lemma, $\chi(h(M)) \leq \chi(M)$. But, since $h(M)$ contains $M$ isometrically, $\chi(M) \leq \chi(h(M))$, which clearly implies the first part of the conclusion. The second part is a direct consequence of Proposition 5.9.

\section{FiXed POINT PROPERTy AND HyperconveXity}

Sine [54] and Soardi [57] results are at the origin of the recent interest to hyperconvex metric spaces. Both Sine and Soardi showed that nonexpansive mappings defined on a bounded hyperconvex metric space have fixed points. Their results were stated in different context but the underlying spaces are simply hyperconvex spaces. Here we will give the proof based on Penot's [49] formulation of Kirk's fixed point theorem. 
Recall that if $T: M \rightarrow M$ is a map, then $x \in M$ is a fixed point of $T$ if $T(x)=x$.

Theorem 6.1. Let $H$ be a bounded hyperconvex metric space. Any nonexpansive map $T: H \rightarrow H$ has a fixed point. Moreover, the fixed point set of T, Fix $(T)$, is hyperconvex.

Proof. Consider $\mathcal{A}(H)$ the family of admissible subsets of $H$. Set

$$
\mathcal{F}=\{A \in \mathcal{A}(H) ; \text { with } A \neq \emptyset \text { and } T(A) \subset A\} .
$$

Obviously, we have $H \in \mathcal{F}$. Since the intersection of any family of nonempty elements of $\mathcal{A}(H)$ is not empty and belongs to $\mathcal{A}(H)$ (because of the hyperconvexity of $H$ ), then $\mathcal{F}$ satisfies Zorn's assumptions. So it has minimal elements. Let $A_{0}$ be one of them. Note that $\operatorname{cov}\left(T\left(A_{0}\right)=A_{0}\right.$. Indeed, since $T\left(A_{0}\right) \subset A_{0}$ and $\operatorname{cov}\left(T\left(A_{0}\right)\right)$ is the smallest admissible set which contains $T\left(A_{0}\right)$, we have $\operatorname{cov}\left(T\left(A_{0}\right)\right) \subset A_{0}$. Using this inclusion, we get

$$
T\left(\operatorname{cov}\left(T\left(A_{0}\right)\right)\right) \subset T\left(A_{0}\right) \subset \operatorname{cov}\left(T\left(A_{0}\right)\right) .
$$

This clearly implies that $\operatorname{cov}\left(T\left(A_{0}\right)\right) \in \mathcal{F}$. The minimality of $A_{0}$ will then imply $\operatorname{cov}\left(T\left(A_{0}\right)\right)=$ $A_{0}$. Now note that $C\left(A_{0}\right)$ belongs to $\mathcal{F}$. Indeed, we know that $C\left(A_{0}\right)$ is not empty and it is in $\mathcal{A}(H)$ because

$$
C\left(A_{0}\right)=\bigcap_{x \in A_{0}} B\left(x, R\left(A_{0}\right)\right) .
$$

Let $x \in C\left(A_{0}\right)$. Then we have $A_{0} \subset B\left(x, R\left(A_{0}\right)\right)$. Since $T$ is nonexpansive, we get $T\left(A_{0}\right) \subset$ $B\left(T(x), R\left(A_{0}\right)\right)$, which implies

$$
A_{0}=\operatorname{cov}\left(T\left(A_{0}\right)\right) \subset B\left(T(x), R\left(A_{0}\right)\right) .
$$

Hence $T(x) \in C\left(A_{0}\right)$. In other words, $C\left(A_{0}\right)$ is invariant under the action of $T$. So we have $C\left(A_{0}\right) \in \mathcal{F}$. Our claim is therefore proved. The minimality of $A_{0}$ will then imply $A_{0}=C\left(A_{0}\right)$. But we have seen that in hyperconvex metric spaces this is not possible for subsets with more than one point. This forces $A_{0}$ to have one point which is a fixed point for $T$. In order to finish the proof of our theorem, we need to show that $\operatorname{Fix}(T)$ is hyperconvex. Let $\left\{x_{i}\right\}_{i \in I}$ be a collection of points in $\operatorname{Fix}(T)$ such that

$$
d\left(x_{i}, x_{j}\right) \leq r_{i}+r_{j}, \text { for any } i, j \in I,
$$

for some positive numbers $\left\{r_{i}\right\}_{i \in I}$. Set $H_{0}=\bigcap_{i \in I} B\left(x_{i}, r_{i}\right)$. The hyperconvexity of $H$ implies that $H_{0}$ is not empty. Since the centers are in $\operatorname{Fix}(T)$ and $T$ is nonexpansive, then we have $T\left(H_{0}\right) \subset H_{0}$. Moreover $H_{0}$ is a bounded hyperconvex metric space, so the above proof implies that $T$ has a fixed point in $H_{0}$, which implies

$$
\operatorname{Fix}(T) \bigcap\left[\bigcap_{i \in I} B\left(x_{i}, r_{i}\right)\right] \neq \emptyset .
$$

This completes the proof of our theorem.

This result is quite amazing. Indeed, if we translate it into the hyperconvex Banach space $l_{\infty}$, we have, for example, that any nonexpansive mapping which leaves a ball invariant has a fixed point. Note that this space is quite bad from a geometrical point of view. It is also a universal space for separable Banach spaces. In other words, any separable Banach space sits inside $l_{\infty}$ isometrically. So for the classical fixed point property this space is very bad. And with this 
theorem, we have a positive fixed point result.

Note that since $\operatorname{Fix}(T)$ is hyperconvex, then any commuting nonexpansive maps $T_{i}, i=$ $1,2, \ldots, n$, defined on a bounded hyperconvex set $H$, have a common fixed point. Moreover their common fixed point set $\operatorname{Fix}\left(T_{1}\right) \cap \operatorname{Fix}\left(T_{2}\right) \cap \cdots \cap \operatorname{Fix}\left(T_{n}\right)$ is hyperconvex.

Combining these results with Baillon's theorem, we get the following:

Theorem 6.2. Let $H$ be a bounded hyperconvex metric space. Any commuting family of nonexpansive maps $\left\{T_{i}\right\}_{i \in I}$, with $T_{i}: H \rightarrow H$, has a common fixed point. Moreover, the common fixed point set $\bigcap_{i \in I} \operatorname{Fix}\left(T_{i}\right)$ is hyperconvex.

Proof. Let $\Gamma=2^{I}=\{\beta ; \beta \subset I\}$. It is obvious that $\Gamma$ is downward directed (the order on $\Gamma$ is the

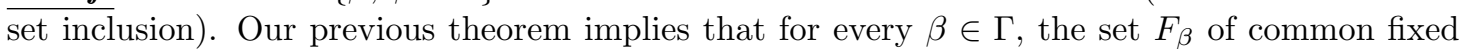
point set of the mappings $T_{i}, i \in \beta$, is nonempty and hyperconvex. Clearly the family $\left(F_{\beta}\right)_{\beta \in \Gamma}$ is decreasing. Using Baillon's result, we deduce that

$$
\bigcap_{i \in I} \operatorname{Fix}\left(T_{i}\right)=\bigcap_{\beta \in \Gamma} F_{\beta}
$$

is nonempty and hyperconvex. The proof is therefore complete.

Remark 6.3. Baillon asked whether boundedness may be relaxed. He precisely asked whether the conclusion holds if the nonexpansive map has a bounded orbit. In the classical Kirk's fixed point theorem, having a bounded orbit implies the existence of a fixed point. Prus answered this question in the negative. Indeed, consider the hyperconvex Banach space $H=l_{\infty}$ and the map $T: H \rightarrow H$ defined by

$$
T\left(\left(x_{n}\right)\right)=\left(1+\lim _{\mathcal{U}} x_{n}, x_{1}, x_{2}, \ldots\right)
$$

where $\mathcal{U}$ is a nontrivial ultrafilter on the set of positive integers. We may also take a Banach limit instead of a limit over an ultrafilter. The map $T$ is an isometry and has no fixed point. On the other hand, we have

$$
T^{n}(0)=(1,1, \ldots, 1,0,0, \ldots)
$$

where the first block of length $n$ has all its entries equal to 1 and 0 after that. So $T$ has bounded orbits. This problem has been studied in $[35,36,40]$.

Recently, we wondered whether this result holds for asymptotically nonexpansive mappings. Recall that a map $T$ is said to be asymptotically nonexpansive if

$$
d\left(T^{n}(x), T^{n}(y)\right) \leq \lambda_{n} d(x, y)
$$

and $\lim _{n} \lambda_{n}=1$. This question is till unknown. But a partial positive answer is known for approximate fixed points. Before we state this result, recall that if $T: H \rightarrow H$ is a map, then $x \in H$ is an $\varepsilon$-fixed point if $d(x, T(x)) \leq \varepsilon$ where $\varepsilon \geq 0$. The set of $\varepsilon$-fixed points of $T$ is denoted by $\operatorname{Fix}_{\varepsilon}(T)$. Sine [55] obtained the following wonderful result:

Theorem 6.4. Let $H$ be a bounded hyperconvex metric space and $T: H \rightarrow H$ a nonexpansive map. For any $\varepsilon>0, F_{i}(T)$ is not empty and is hyperconvex. 
Proof. $\operatorname{Fix}_{\varepsilon}(T)$ is nonempty since $T$ has fixed point. Let $\left\{x_{\alpha}\right\}_{\alpha \in \Gamma}$ be points in $\operatorname{Fix}_{\varepsilon}(T)$ and $\overline{\left\{r_{\alpha}\right\}_{\alpha \in \Gamma}}$ be positive numbers such that $d\left(x_{\alpha}, x_{\beta}\right) \leq r_{\alpha}+r_{\beta}$ for any $\alpha, \beta \in \Gamma$. Set

$$
J=\bigcap_{\alpha \in \Gamma} B\left(x_{\alpha}, r_{\alpha}\right) .
$$

We know that $J$ is nonempty as a subset of $H$. We wish to show that $J \cap F x_{\varepsilon}(T)$ is not empty. Let $x \in J$. Then $T(x) \in J+\varepsilon$ because $d\left(T x, x_{\alpha}\right) \leq d\left(T x, T x_{\alpha}\right)+d\left(T x_{\alpha}, x_{\alpha}\right) \leq d\left(x, x_{\alpha}\right)+\varepsilon \leq r_{\alpha}+\varepsilon$ for any $\alpha$. Using Theorem 4.12, there exists a nonexpansive retraction $\Pi: J+\varepsilon \rightarrow \bar{J}$ which is $\varepsilon$-constant. The map $R: J \rightarrow J$ defined by $R(x)=\Pi \circ T(x)$ is nonexpansive. Since $J$ is a bounded hyperconvex metric space, then $R$ has a fixed point $x_{0} \in J$. Since $\Pi$ is $\varepsilon$-constant, we get

$$
d\left(x_{0}, T\left(x_{0}\right)\right)=d\left(\Pi \circ T\left(x_{0}\right), T\left(x_{0}\right)\right) \leq \varepsilon
$$

which implies that $J \cap \operatorname{Fix}_{\varepsilon}(T) \neq \emptyset$. The proof is therefore complete.

Now we are ready to state the following unpublished result.

Theorem 6.5. Let $H$ be a bounded hyperconvex metric space and $T: H \rightarrow H$ be asymptotically nonexpansive. For any $\varepsilon>0, F i x_{\varepsilon}(T)$ is not empty, in other words we have

$$
\inf _{x \in H} d(x, T(x))=0 .
$$

Proof. Using the convexity shown in Remark 4.9, we define

$$
T_{n}(x)=\left(1-\frac{1}{\lambda_{n}}\right) a \oplus \frac{1}{\lambda_{n}} T^{n}(x)
$$

where $a$ is a fixed point in $H, \lambda_{n}$ is the Lipschitz constant of $T^{n}$. The maps $T_{n}$ are nonexpansive. Before we proceed with the proof we need to define the "ultrapower" of $H$. Consider the cartesian product $\mathcal{H}=\prod_{n \geq 1} H$, and let $\mathcal{U}$ be a nontrivial ultrafilter on the natural numbers. Define the equivalence relation $\sim$ on $\mathcal{H}$ by $\left(x_{n}\right) \sim\left(y_{n}\right)$ if and only if $\lim _{\mathcal{U}} d\left(x_{n}, y_{n}\right)=0$. The limit over $\mathcal{U}$ exists since $H$ is bounded. Then we consider the quotient set $\widetilde{H}$. An element $\tilde{x} \in \widetilde{H}$ is a subset of $\mathcal{H}$. If $\left(x_{n}\right) \in \tilde{x}$, then $\left(y_{n}\right) \in \tilde{x}$ if and only if $\lim _{\mathcal{U}} d\left(x_{n}, y_{n}\right)=0$. On $\widetilde{H}$ define the metric $\tilde{d}$ by $\tilde{d}(\tilde{x}, \tilde{y})=\lim _{\mathcal{U}} d\left(x_{n}, y_{n}\right)$ where $\left(x_{n}\right)$ (resp. $\left.\left(y_{n}\right)\right)$ is any element in $\tilde{x}$ (resp. $\tilde{y}$ ). It is easy to see that $\widetilde{H}$ endowed with the distance $\tilde{d}$ has many nice properties similar to the linear ultrapower of a Banach space. Define the operators $\hat{T}$ and $\tilde{T}$ by

$$
\hat{T}(\tilde{x})=\hat{T}\left(\widetilde{\left(x_{n}\right)}\right)=\left(\widetilde{T^{n}\left(x_{n}\right)}\right) \text { and } \tilde{T}(\tilde{x})=\tilde{T}\left(\widetilde{\left(x_{n}\right)}\right)=\left(\widetilde{T\left(x_{n}\right)}\right) \text {. }
$$

Since $T$ is asymptotically nonexpansive, the operator $\hat{T}$ is nonexpansive. Moreover we have $\hat{T}\left(\widetilde{\left(x_{n}\right)}\right)=\left(\widetilde{T_{n}\left(x_{n}\right)}\right)$. Since $T_{n}$ is nonexpansive, it has a fixed point $x_{n}$. The point $\tilde{x}=\widetilde{\left(x_{n}\right)}$ is a fixed point of $\hat{T}$. Hence the fixed point set $\operatorname{Fix}(\hat{T})$ is a nonempty subset of $\widetilde{H}$. Since the two operators $\hat{T}$ and $\tilde{T}$ commute, then $\tilde{T}$ leaves invariant the set $\operatorname{Fix}(\hat{T})$. It is easy to show that $\tilde{T}$ restricted to $\operatorname{Fix}(\hat{T})$ is in fact an isometry (in particular it is nonexpansive). Fix $\varepsilon>0$. Let $\tilde{x}_{i} \in \operatorname{Fix}(\hat{T}), i=1, \ldots, N$. Set

$$
\varepsilon_{n}=\max _{1 \leq i \leq N} d\left(x_{n}(i), T_{n}\left(x_{n}(i)\right)\right)
$$

for $n \geq 1$, where $\tilde{x}_{i}=\widetilde{\left(x_{n}(i)\right)}$. Set

$$
H_{n}=\left\{x \in H ; d\left(x, T_{n}(x)\right) \leq \varepsilon_{n}\right\} .
$$


Hence $x_{n}(i) \in H_{n}$, for $i=1, \ldots, N$ and any $n \geq 1$. Since $T_{n}$ is nonexpansive, Theorem 6.4 implies that $H_{n}$ is hyperconvex. Therefore, there exist $z_{n}(i)=\varepsilon x_{n}(1) \oplus(1-\varepsilon) x_{n}(i) \in H_{n}$ for $i=1, \ldots, N$. Consider, the point $\tilde{z}_{i}=\widehat{\left(z_{n}(i)\right)}$, which we will denote $\varepsilon \tilde{x}_{1} \oplus(1-\varepsilon) \tilde{x}_{i}$. Then we have, $\tilde{z}_{i} \in \operatorname{Fix}(\hat{T})$, and $\tilde{d}\left(\tilde{z}_{i}, \tilde{z}_{j}\right) \leq(1-\varepsilon) d\left(\tilde{x}_{i}, \tilde{x}_{j}\right)$ for $i, j=2, \ldots, N$. Back to our maps $\hat{T}$ and $\tilde{T}$, let $\tilde{x} \in \operatorname{Fix}(\hat{T})$, and write $\tilde{x}=\tilde{x}_{1}$. Set

$$
\tilde{x}_{2}=\varepsilon \tilde{x}_{1} \oplus(1-\varepsilon) \tilde{T}\left(\tilde{x}_{1}\right) .
$$

Then $\tilde{x}_{2} \in \operatorname{Fix}(\hat{T})$. By induction, we will construct a sequence $\left(\tilde{x}_{n}\right)$ of points in $\operatorname{Fix}(\hat{T})$ defined by $\tilde{x}_{n+1}=\varepsilon \tilde{x}_{1} \oplus(1-\varepsilon) \tilde{T}\left(\tilde{x}_{n}\right)$. For any $n<m$, we have

$$
d\left(\tilde{x}_{n}, \tilde{x}_{m}\right) \leq(1-\varepsilon) d\left(\tilde{T}\left(\tilde{x}_{n-1}\right), \tilde{T}\left(\tilde{x}_{m-1}\right)\right) .
$$

Since $\tilde{T}$ is nonexpansive when restricted to $\operatorname{Fix}(\hat{T})$, we get

$$
d\left(\tilde{x}_{n}, \tilde{x}_{m}\right) \leq(1-\varepsilon) d\left(\tilde{x}_{n-1}, \tilde{x}_{m-1}\right) .
$$

This clearly implies that the sequence $\left(\tilde{x}_{n}\right)$ is a Cauchy sequence. Hence it converges to $\tilde{\omega} \in$ $\operatorname{Fix}(\hat{T})$. Moreover we have

$$
d(\tilde{\omega}, \tilde{T}(\tilde{\omega}))=\lim _{n \rightarrow \infty} d\left(\tilde{x}_{n+1}, \tilde{T}\left(\tilde{x}_{n}\right)\right) \leq \varepsilon \lim _{n \rightarrow \infty} d\left(\tilde{x}_{1}, \tilde{T}\left(\tilde{x}_{n}\right)\right)
$$

If we set $\delta=\operatorname{diameter}(H)$, we get $d(\tilde{\omega}, \tilde{T}(\tilde{\omega})) \leq \varepsilon \delta$, so for any $\varepsilon>0$ there exists $\tilde{\omega}_{\varepsilon} \in \operatorname{Fix}(\hat{T})$ such that $d\left(\tilde{\omega}_{\varepsilon}, \tilde{T}\left(\tilde{\omega}_{\varepsilon}\right)\right) \leq \varepsilon$. Classical argument implies that for any $\varepsilon>0$ there exists $x_{\varepsilon} \in H$ such that $d\left(x_{\varepsilon}, T\left(x_{\varepsilon}\right)\right) \leq \varepsilon$ which completes the proof of Theorem 6.5.

\section{TOPOlOGiCAl FiXed POINT THEOREMS AND HyperCONVEXity}

Another important branch of fixed point theory is that one formed by those results in which topological-like conditions are considered. We may think of the well-known Schauder theorem as the starting point of this branch. This theorem states that any mapping defined from a nonempty compact and convex subset of a Banach space into itself must have a fixed point. An easy improvement of this theorem is obtained when the compactness condition is imposed on the mapping instead of on its domain. New achievements came when the compactness condition on the mapping was treated in more general terms. Let $M$ be a metric space and let $\mathcal{B}(M)$ be the collection of nonempty, and bounded subsets of $M$, then a mapping $\gamma: \mathcal{B}(M) \rightarrow[0,+\infty)$ is called a measure of noncompactness if it satisfies the following conditions:

(1) $\gamma(A)=0$ if and only if $A$ is precompact.

(2) $\gamma(A)=\gamma(\bar{A})$ for any $A \in \mathcal{B}(M)$.

(3) $\gamma(A \cup B)=\max \{\gamma(A), \gamma(B)\}$ for any $A, B \in \mathcal{B}(M)$.

Of course, as it was announced in Section 5, the mappings $\alpha$ and $\chi$ given by Definition 5.8 are measures of noncompactness. A new kind of mapping arises naturally.

Definition 7.1. Let $M$ be a metric space and $D \subseteq M$. A mapping $T: D \rightarrow M$ is said to be a $\gamma$-condensing (or condensing relative to $\gamma$ ) mapping if $T$ is continuous and if for each bounded $A \subseteq D$, for which $\gamma(A)>0, \gamma(T(A))<\gamma(A)$. 
A detailed studied of these mappings may be found in [2]. It is easy to see, however, that any compact mapping is condensing relative to any measure $\gamma$. The also well-known DarboSadovskii's theorem [52] states that if $\gamma$ is a measure of noncompactness defined on a normed space such that $\gamma(B)=\gamma(\operatorname{conv}(B))$ for any nonempty and bounded subset of the normed space, and $T$ is a $\gamma$-condensing mapping from a nonempty bounded closed and convex subset of the normed space into itself, then $T$ has a fixed point. The condition $\gamma(B)=\gamma(\operatorname{conv}(B))$ of invariance when convex hull is considered is fundamental for this result and other related which hyperconvex counterparts will seen below. Measures of Kuratowski and Hausdorff studied in Section 5 are among those that satisfy the above condition. Even more, Corollary 5.11 says that both measures satisfy an equivalent condition for hyperconvex spaces. We will make use of it later in this section. Surprisingly, as it was noted in [40], a hyperconvex version of Darbo-Sadovskii theorem does not require of that corollary.

Theorem 7.2. Let $H$ be a bounded hyperconvex space and $T: H \rightarrow H$ a $\alpha$-condensing mapping. Then $T$ has a fixed point.

Proof. From Section 3 we may assume that $H$ is a bounded closed subset of a Banach space. We also know that there exists a nonexpansive retraction $R: \operatorname{cl}-\operatorname{conv}(H) \rightarrow H$ (where $\operatorname{cl}-\operatorname{conv}(H)$ denotes the closed convex hull of $H)$. Then $T \circ R: \operatorname{cl}-\operatorname{conv}(H) \rightarrow H$, and if $A \subseteq \operatorname{cl}-\operatorname{conv}(H)$ satisfies that $\alpha(A)>0$ then either $\alpha(R(A))=0$ or

$$
\alpha(T \circ R(A))<\alpha(R(A)) \leq \alpha(A) .
$$

In either case $\alpha(T \circ R(A))<\alpha(A)$ so $T \circ R$ is also $\alpha$-condensing. Now Darbo-Sadovskii theorem implies the existence of a fixed point $x$ for $T \circ R$. It is easy to see that it must also be a fixed point for $T$.

Remark 7.3. Notice that $\alpha$ may be replaced by any measure of noncompactness for which DarboSadovskii theorem holds.

The classes of condensing operators defined relative to distinct measure of noncompactness are not equal in general, but they nevertheless share a number of general properties. Bearing in mind these common properties Sadovskii [53] introduced the concept of limit operator without using the notion of measure of noncompactness. If $X$ is a linear space and $D$ is a subset of $M$, then a continuous operator $T: D \rightarrow X$ is called a limit compact or ultimately compact operator if cl-conv $(T(B \cap D))=B$, for $B \subseteq X$, implies that $B$ is compact. The concept of hyperconvex hull will help us to define limit compact operators in hyperconvex spaces.

Definition 7.4. Let $D$ be a subset of a hyperconvex metric space $H$. Given an operator $T: D \longrightarrow$ $H$ we will say that $\left(T_{\alpha}\right)$ is a transfinite sequence associated to $T$ on $D$ if

(1) $T_{0}=h(T(D))$

(2) $T_{\alpha}=h\left(T\left(D \cap T_{\alpha-1}\right)\right)$ if $\alpha-1$ exists,

(3) $T_{\alpha}=\bigcap_{\beta<\alpha} T_{\beta}$, if $\alpha-1$ does not exist,

where $T_{\alpha}$ is a hyperconvex hull of $T(D)$ or $T\left(D \cap T_{\alpha-1}\right)$ so that the sequence $\left(T_{\alpha}\right)$ is nonincreasing.

Remark 7.5. It is easy to deduce from the properties of the hyperconvex hull that given $D, M$ and $f$ as in the previous definition, there always exists a transfinite sequence associated to $T$ on $D$.

The proof of the following lemma is rather easy, we will omit it. 
Lemma 7.6. If $\left(T_{\alpha}\right)$ is a transfinite sequence associated to $T$ on $D$, then:

(1) Each $T_{\alpha}$ is closed.

(2) $T\left(D \cap T_{\alpha}\right) \subseteq T_{\alpha+1}$ for all $\alpha$.

(3) If $\beta<\alpha$, then $T_{\alpha} \subseteq T_{\beta}$.

(4) $T\left(D \cap T_{\alpha}\right) \subseteq T_{\alpha}$ for all $\alpha$.

(5) There exists an ordinal number $\eta$ such that $T_{\alpha}=T_{\eta}$ for all $\alpha \geq \eta$.

Hence we obtain the following corollary.

Corollary 7.7. Let $\left(T_{\alpha}\right)$ be a transfinite sequence associated to $T$ on $H$ (hyperconvex), and suppose $H$ is bounded. Then the set $T_{\eta}$ given by Lemma 7.6 is nonempty.

Proof. It suffices to show that for any ordinal number $\alpha, T_{\alpha} \neq \emptyset$. We proceed by transfinite induction. The case $\alpha=0$ is trivial since $T(D) \subseteq T_{0}$, and therefore $T_{0}$ is nonempty. Now we have to consider the following two cases: the ordinal $\alpha$ has a predecessor, in which case, from the inductive hypothesis, $T_{\alpha-1}$ is nonempty and hence, $T_{\alpha}=h\left(T\left(M \cap T_{\alpha-1}\right)\right)$ is nonempty, or the ordinal $\alpha$ has no predecessor, in which case $T_{\alpha}=\bigcap_{\beta<\alpha} T_{\beta}$. By using the inductive hypothesis and Baillon's intersection result (Theorem 5.1), it follows that $T_{\alpha}$ is nonempty.

This corollary allows us to define the concept of limit compact operator in hyperconvex spaces.

Definition 7.8. We say that $T^{\infty}(D)$ is an ultimate range of the operator $T$ on the set $H$ if it is the limit set of a transfinite sequence associated to $T$ on $D$. The operator $T$ is said to be ultimately compact (or limit compact) if is continuous and there exists an ultimate range $T^{\infty}(D)$ of $T$ such that $T\left(D \cap T^{\infty}(D)\right)$ is relatively compact on $H$.

We offer the following lemma without proof, for details see [16].

Lemma 7.9. The following properties hold:

(1) $T^{\infty}(D)=h\left(T\left(D \cap T^{\infty}(D)\right)\right)$.

(2) If $D_{1} \subseteq D$, then $T: D_{1} \rightarrow H$ has an ultimate range $T^{\infty}\left(D_{1}\right)$ contained in $T^{\infty}(D)$.

(3) If $T$ is ultimately compact on $D$ and $D_{1} \subseteq D, T$ is ultimately compact on $D_{1}$.

(4) The operator $T: D \rightarrow H$ is ultimately compact if and only if $T^{\infty}(D)$ is compact.

(5) The operator $T: D \rightarrow H$ is ultimately compact if and only if for any $B \subset H$, the equality $h(f(B \cap D))=B$ implies that $B$ is compact.

The following theorem states the relation on $\alpha$-condensing and limit compact mappings.

Theorem 7.10. Let $H$ be a bounded hyperconvex set, and suppose $D \subseteq H$ is closed. If $T: D \rightarrow H$ is $\alpha$-condensing, then $T$ is ultimately compact on $D$.

Proof. Let $T^{\infty}(D)$ be any ultimate range of $T$ on $D$. By the previous lemma $h\left(T\left(D \cap T^{\infty}(D)\right)\right)=$ $\overline{T^{\infty}(D)}$. From this, $h\left(T\left(D \cap T^{\infty}(D)\right)\right) \supseteq D \cap T^{\infty}(D)$. Since $\alpha$ is monotonous, we obtain $\alpha(h(T(D \cap$ $\left.\left.T^{\infty}(D)\right)\right) \geq \alpha\left(D \cap T^{\infty}(D)\right)$. But, from Corollary 5.11, $\alpha\left(h\left(T\left(D \cap T^{\infty}(D)\right)\right)=\alpha\left(T\left(D \cap T^{\infty}(D)\right)\right)\right.$. And hence $\alpha\left(T\left(D \cap T^{\infty}(D)\right)\right) \geq \alpha\left(D \cap T^{\infty}(D)\right)$. Bearing in mind that $T$ is condensing we conclude $D \cap T^{\infty}(D)$ is relatively compact. Therefore $T$ is ultimately compact on $D$.

The following theorem states the existence of fixed point for limit compact mappings, so, in view of the previous theorem, it may be understood as an extension of Theorem 7.2.

Theorem 7.11. Let $H$ be a bounded hyperconvex metric space and $T: H \rightarrow H$ a limit compact mapping on $H$. Then $T$ has a fixed point in $H$. 
Proof. The conclusion follows from Schauder theorem since $T^{\infty}(H)$ is nonempty, compact and hyperconvex, and

$$
T: T^{\infty}(M) \longrightarrow T^{\infty}(M) \text {. }
$$

The following theorem was stated in [5] and it is related to the previous one although, as it was seen in [4], they are different.

Theorem 7.12. Let $H$ be a hyperconvex metric space, $x_{0} \in H$, and let $T$ be a continuous mapping from $H$ into itself. If for every $D \subseteq H$ such that $D$ is isometric to $h(T(D))$ or $D=T(D) \cup\left\{x_{0}\right\}$ then $D$ is relatively compact, then $T$ has a fixed point in $M$.

Proof. The first part of the proof consists in proving that there exists $Z \subseteq H$ such that $T(Z)=Z$. $\overline{\text { Let } \Sigma}=\left\{U \subseteq H: U\right.$ is $T$-invariant, and $\left.x_{0} \in U\right\} . \Sigma$ is nonempty since $H$ belongs to it. It is easy to see that

$$
B=\bigcap_{U \in \Sigma} U
$$

is a nonempty, and minimal among all the elements of $\Sigma$, i.e. it does not contain any proper subset $V$ such that $T(V) \cup\left\{x_{0}\right\} \subseteq V$. From here it is possible to prove that $B$ satisfies that $T(B) \cup\left\{x_{0}\right\}=B$ and hence, by hypothesis, that $B$ is relatively compact. We will omit details that may be found in [58], this implies the existence of the set $Z$.

Let $\Omega$ be the family of all sets $A \subseteq H$ such that $Z \subseteq A, A$ is hyperconvex and $f(A) \subseteq A$. $\Omega$ is nonempty since $H$ belongs to it. Zorn's lemma leads to a minimal element in $\Omega$, let us say $H_{0}$. So $T\left(H_{0}\right) \subseteq H_{0}$, and, since $H_{0}$ is hyperconvex, there exists a hyperconvex hull $h\left(T\left(H_{0}\right)\right)$ of $T\left(H_{0}\right)$ such that $T\left(H_{0}\right) \subseteq h\left(T\left(H_{0}\right)\right) \subseteq H_{0}$. Moreover we have $T\left(h\left(T\left(H_{0}\right)\right)\right) \subseteq T\left(H_{0}\right) \subseteq h\left(T\left(H_{0}\right)\right)$ and $Z=T(Z) \subseteq T\left(H_{0}\right) \subseteq h\left(T\left(H_{0}\right)\right)$. Hence, since $h\left(T\left(H_{0}\right)\right)$ is hyperconvex, we have $h\left(T\left(H_{0}\right)\right) \in \Omega$. Now, from the minimality of $H_{0}$, we obtain $h\left(T\left(H_{0}\right)\right)=H_{0}$ and so, from hypothesis, $H_{0}$ is relatively compact but additionally $H_{0}$ is hyperconvex, and hence closed, which implies that $H_{0}$ is compact. The conclusion follows since $T: H_{0} \rightarrow H_{0}$ is a continuous compact mapping and $H_{0}$ is hyperconvex.

Remark 7.13. It is not difficult to see that any $\alpha$-condensing mapping satisfies the hypothesis of this theorem. Notice also that $H$ is not required to be bounded in this theorem.

We finish this section with the following theorem stated in [40].

Theorem 7.14. Let $H$ be a bounded hyperconvex metric space. Let $T: H \rightarrow H$, and suppose for some relatively prime pair $i, j \in \mathbb{N}, T^{i}$ is nonexpansive and $T^{j}$ is condensing. Then $T$ has a fixed point.

Proof. Let $A=\left\{n \in \mathbb{N}: T^{n}\right.$ is either nonexpansive or condensing $\}$. Since $A$ is additive, there exists an integer $N$ such that if $n \geq N$ then $n \in A$. It follows that there exist $n$ and $n+1$ such that $T^{n}$ is nonexpansive and $T^{n+1}$ is condensing. Since $T^{n}$ and $T^{n+1}$ commute, and $\operatorname{Fix}\left(T^{n}\right)$ is hyperconvex, as it was shown in Section $6, T^{n+1}$ must have a common fixed point with $T^{n}$. This point is also a fixed point for $T$.

Remark 7.15. It is an interesting open question whether the previous theorem still holds if the nonexpansiveness condition is removed. 


\section{Isbell's Hyperconvex Hull}

As we mentioned before, Isbell [24] showed that every metric space has an injective envelope. The injective envelope of a metric space $M$ is an injective metric space $\tilde{M}$ which contains an isometric copy of $M$ and which is isometric with a subspace of any hyperconvex metric space which contains an isometric copy of $M$. In this section, we will discuss Isbell's ideas.

Let $M$ be a metric space. For any $x \in M$, define the positive real valued function $f_{x}: M \rightarrow$ $[0, \infty)$ by $f_{x}(y)=d(x, y)$. Let us discuss some of the properties verified by these functions.

1. Using the triangle inequality, we get

$$
d(x, y) \leq f_{a}(x)+f_{a}(y)
$$

and

for any $x, y, a \in M$.

$$
f_{a}(x) \leq d(x, y)+f_{a}(y)
$$

2. Let $f: M \rightarrow[0, \infty)$ be such that $d(x, y) \leq f(x)+f(y)$, for any $x, y \in M$, and for some $a \in M$, assume that $f(x) \leq f_{a}(x)$, for any $x \in M$. In this case, we have $f=f_{a}$. Indeed, first we have $f(a) \leq f_{a}(a)=0$, which implies $f(a)=0$. Using the above inequality, we get

$$
f_{a}(x)=d(x, a) \leq f(x)+f(a)=f(x)
$$

for any $x \in M$. Combined with the assumptions on $f(x)$, we get $f(x)=f_{a}(x)$, for any $x \in M$. This is a minimality property for the pointwise order.

Using these properties, Isbell introduced what he called the injective envelope of $A$, denoted $\epsilon(A)$, for any subset $A$ of $M$. The set $\epsilon(A)$ is the set of all extremal functions defined on $A$. Recall that the function $f: A \rightarrow[0, \infty)$ is extremal if $d(x, y) \leq f(x)+f(y)$ for all $x, y$ in $A$ and is pointwise minimal, i.e. if $g: A \rightarrow[0, \infty)$ such that $d(x, y) \leq g(x)+g(y)$ for all $x, y$ in $A$ and $g(x) \leq f(x)$ for all $x \in A$, then we must have $f=g$. In particular, we have $f_{a} \in \epsilon(A)$, for any $a \in A$. Consider the map $e: A \rightarrow \epsilon(A)$, defined by $e(a)=f_{a}$, for all $a \in A$. The map $e$ is an isometry. In other words, we have

$$
d(e(a), e(b))=\sup _{x \in A}\left|f_{a}(x)-f_{b}(x)\right|=\sup _{x \in A}|d(a, x)-d(b, x)|=d(a, b) .
$$

So, $A$ and $e(A)$ are isometric spaces, hence we may identify $A$ and $e(A)$, or $a \in A$ with $e(a)$. Before we give some detailed properties of extremal functions, we will need the following easy to prove lemma.

Lemma 8.1. Let $A$ be a nonempty subset of $M$. Let $r: A \rightarrow[0, \infty)$ be such that

$$
d(x, y) \leq r(x)+r(y)
$$

for any $x, y \in A$. Then there exits $R: M \rightarrow[0, \infty)$ which extends $r$ such that

$$
d(x, y) \leq R(x)+R(y)
$$

for any $x, y \in M$. Moreover, there exists an extremal function $f$ defined on $M$ such that $f(x) \leq R(x)$ for any $x \in M$.

Let us give some properties of extremal functions.

Proposition 8.2. The following statements are true. 
1. If $f \in \epsilon(A)$, then it satisfies $f(x) \leq d(x, y)+f(y)$ for all $x, y$ in $A$. Moreover, we have

$$
f(x)=\sup _{y \in A}\left|f(y)-f_{x}(y)\right|=d(f, e(x)) .
$$

2. For any $f \in \epsilon(A), \delta>0$, and $x \in A$, there exists $y \in A$ such that

$$
f(x)+f(y)<d(x, y)+\delta .
$$

3. If $A$ is compact, then $\epsilon(A)$ is compact.

4. If $s$ is an extremal function on the metric space $\epsilon(A)$, then $s \circ e$ is extremal on $A$.

Proof. 1. Assume not. Then there exist $x_{0}, y_{0} \in A$, such that

$$
d\left(x_{0}, y_{0}\right)+f\left(y_{0}\right)<f\left(x_{0}\right) .
$$

Set

$$
g(x)= \begin{cases}f(x) & \text { if } x \neq x_{0} \\ d\left(x_{0}, y_{0}\right)+f\left(y_{0}\right) & \text { if } x=x_{0}\end{cases}
$$

It is clear that we have $g(x) \leq f(x)$, for any $x \in A$. In particular, we have $g\left(x_{0}\right)<f\left(x_{0}\right)$. Let us show that for any $x, y \in A$, we have $d(x, y) \leq g(x)+g(y)$. If both $x$ and $y$ are different from $x_{0}$, we use the properties of $f$. So we can assume $x=x_{0}$ and $y \neq x_{0}$. Then

$$
d(x, y)=d\left(x_{0}, y\right) \leq d\left(x_{0}, y_{0}\right)+d\left(y_{0}, y\right) \leq d\left(x_{0}, y_{0}\right)+f\left(y_{0}\right)+f(y),
$$

which obviously implies $d\left(x_{0}, y\right) \leq g\left(x_{0}\right)+g(y)$. The minimality behavior of $f$ gives us $f=g$ which is a contradiction.

Combining this inequality with the fundamental one (for extremal functions), we get $\mid f(y)-$ $f_{x}(y) \mid \leq f(x)$ for any $y \in A$. The equality holds for $y=x$ which clearly implies

$$
f(x)=\sup _{y \in A}\left|f(y)-f_{x}(y)\right|=d(f, e(x)) .
$$

2. Assume not. Then there exist $x \in A$ and $\delta>0$ (we may take it less than $f(x)$ ) such that for any $y \in A$, we have

Set

$$
d(x, y)+\delta \leq f(x)+f(y)
$$

$$
h(z)= \begin{cases}f(z) & \text { if } z \neq x \\ f(x)-\delta & \text { if } z=x .\end{cases}
$$

It is easy to check that $d(y, z) \leq h(y)+h(z)$ for any $y, z \in A$. Since $h \leq f$ and $h(x)<f(x)$, we get a contradiction with the minimality of $f$. This completes the proof of Property 2 .

3. From the property 1 , we get

$$
|f(x)-f(y)| \leq d(x, y)
$$

for any $x, y \in A$. This implies $\epsilon(A) \subset \operatorname{Lip}_{1}(A)$, where $\operatorname{Lip}_{1}(A)$ is the space of all Lipschitzian real valued functions with Lipschitz constant equal 1 . Hence, all extremal functions are equicontinuous. Also, it is quite easy to show that pointwise-limit of extremal functions is an extremal function. Since $A$ is compact, the Arzel -Ascoli theorem implies that $\epsilon(A)$ is compact.

4. Let $s$ be an extremal function on the metric space $\epsilon(A)$. Note that for any $x, y \in A$, we have

$$
d(x, y)=d\left(f_{x}, f_{y}\right) \leq s\left(f_{x}\right)+s\left(f_{y}\right)=s \circ e(x)+s \circ e(y) .
$$

Assume that $s \circ e$ is not an extremal function on $A$. Then there exists an extremal function $h \in \epsilon(A)$ such that $h(x) \leq s \circ e(x)$, for any $x \in A$, and the inequality is strict at some point $x_{0}$. Define the function

$$
t(f)= \begin{cases}s(f) & \text { if } f \neq e\left(x_{0}\right) \\ h\left(x_{0}\right) & \text { if } f=e\left(x_{0}\right) .\end{cases}
$$


Let us show that $t$ satisfies the inequality $d(f, g) \leq t(f)+t(g)$ for any $f, g \in \epsilon(A)$. Since $t$ and $s$ coincide almost everywhere and $s$ is an extremal function, then we only need to prove the above inequality for $g=e\left(x_{0}\right)$ and $f \neq e\left(x_{0}\right)$, i.e. $d\left(f, e\left(x_{0}\right)\right) \leq t(f)+t\left(e\left(x_{0}\right)\right)$.

For any $\delta>0$, there exists $y \in A$ such that $f\left(x_{0}\right)+f(y)<d\left(x_{0}, y\right)+\delta$. If $y=x_{0}$, then we must have $f\left(x_{0}\right) \leq 1 / 2 \delta$. Hence

$$
d\left(f, e\left(x_{0}\right)\right)=f\left(x_{0}\right) \leq \frac{1}{2} \delta+t(f)+t\left(e\left(x_{0}\right)\right) .
$$

On the other hand, if $y \neq x_{0}$ and $f \neq e\left(x_{0}\right)$, then

$$
d\left(f, e\left(x_{0}\right)\right)+f(y)-\delta=f\left(x_{0}\right)+f(y)-\delta<d\left(x_{0}, y\right)
$$

and

$$
d\left(x_{0}, y\right) \leq h\left(x_{0}\right)+h(y) \leq h\left(x_{0}\right)+s \circ e(y)=t\left(e\left(x_{0}\right)\right)+t(e(y)) .
$$

Since $s$ is an extremal function, then

$$
t(e(y))=s(e(y)) \leq s(f)+d(f, e(y))=t(f)+f(y)
$$

where we used the fact that $f$ is an extremal function (to get $d(f, e(y))=f(y)$ ). So we have the two inequalities

$$
d\left(f, e\left(x_{0}\right)\right)+f(y)-\delta<t\left(e\left(x_{0}\right)\right)+t(e(y))
$$

and

$$
t(e(y)) \leq t(f)+f(y)
$$

Adding the two inequalities, we get

$$
d\left(f, e\left(x_{0}\right)\right)+f(y)-\delta+t(e(y))<t\left(e\left(x_{0}\right)\right)+t(e(y))+t(f)+f(y)
$$

which leads to $d\left(f, e\left(x_{0}\right)\right)-\delta<t\left(e\left(x_{0}\right)\right)+t(f)$. Since $\delta$ is arbitrary, we get the desired inequality

$$
d\left(f, e\left(x_{0}\right)\right) \leq t\left(e\left(x_{0}\right)\right)+t(f) .
$$

The link between Isbell's ideas and hyperconvexity is given in the following proposition.

Proposition 8.3. The following statements are true.

1. $\epsilon(A)$ is hyperconvex.

2. $\epsilon(A)$ is an injective envelope of $A$, i.e. no proper subset of $\epsilon(A)$ which contains $A$ (metrically) is hyperconvex.

Proof. 1. In order to prove that $\epsilon(A)$ is hyperconvex, let $\left\{f_{\alpha}\right\}$ be a family of points in $\epsilon(A)$ and

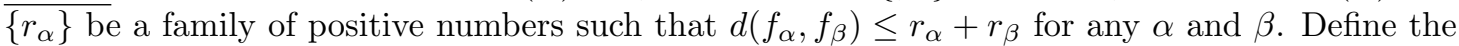
map $r:\left\{f_{\alpha}\right\} \rightarrow[0, \infty)$ by $r\left(f_{\alpha}\right)=r_{\alpha}$. By Lemma 8.1, we extend $r$ to the entire set $\epsilon(A)$ such that $d(f, g) \leq r(f)+r(g)$ for any $f, g \in \epsilon(A)$. Using the same Lemma 8.1, there exists $h$ an extremal function on $\epsilon(A)$ such that $h \leq r$. Using Property 4 of extremal functions, we know that $h \circ e$ is an extremal function on $A$, i.e. $h \circ e \in \epsilon(A)$. It is easy to see that

$$
h \circ e \in \bigcap_{f \in \epsilon(A)} B(f, r(f)) \subset \bigcap_{\alpha} B\left(f_{\alpha}, r_{\alpha}\right) .
$$

Indeed, we have

$$
h \circ e(x)-f(x)=h \circ e(x)-d(f, e(x)) \leq h(f) \leq r(f)
$$

and

$$
f(x)-h \circ e(x)=d(f, e(x))-h \circ e(x) \leq h(f) \leq r(f)
$$

for any $x \in A$. Hence $d(f, h \circ e) \leq r(f)$ for any $f \in \epsilon(A)$. The proof of our claim is therefore complete. 
2. Let $H$ be a subset of $\epsilon(A)$ such that $e(A) \subset H$. Assume that $H$ is hyperconvex. Since $\epsilon(A)$ is hyperconvex, there exists a nonexpansive retraction $R: \epsilon(A) \rightarrow H$. Let $f \in \epsilon(A)$. We have

$$
d(R(f), e(x))=R(f)(x) \leq d(f, e(x))=f(x)
$$

for any $x \in A$. Since $f$ is an extremal function, we must have $R(f)=f$. This clearly implies that $H=\epsilon(A)$. So no proper subset of $\epsilon(A)$ which contains $e(A)$ is hyperconvex.

Remark 8.4. The idea of linear injective envelope for Banach spaces and metric injective envelope were introduced by Cohen [9] and Isbell [24] respectively, both in 1964. Since then many interesting papers came out studying properties of these elements as well as the question whether the metric injective envelope of a normed spaces coincides with its linear one. This problem was finally solved in the affirmative by Rao [51] in 1992. For this and other interesting properties of injective envelopes the reader may consult [7, 9, 10, 21, 24, 25, 26, 51].

\section{Set-Valued mappings in Hyperconvex spaces}

Recall that $\mathcal{A}(M)$ denotes the family of all nonempty admissible subsets of a metric space $M$ and set throughout this section $\mathcal{E}(M)$ as the family of all nonempty bounded subsets of $M$ which are externally hyperconvex (relative to $M$ ). In both instances endowed with the usual Hausdorff metric $d_{H}$. Recall that the distance between two closed subsets $A, B$ of a metric space in the Hausdorff sense is given by

$$
d_{H}(A, B)=\inf \left\{\varepsilon>0: A \subset N_{\varepsilon}(B) \text { and } B \subset N_{\varepsilon}(A)\right\}
$$

where $N_{\varepsilon}(A)$ denotes the closed $\varepsilon$-neighborhood of $A$.

The main result of this section is the following selection theorem stated in [33].

Theorem 9.1. Let $H$ be hyperconvex, and let $T^{*}: H \rightarrow \mathcal{E}(H)$. Then there exists a mapping $T:$ $H \rightarrow H$ for which $T(x) \in T^{*}(x)$ for each $x \in H$ and for which $d(T(x), T(y)) \leq d_{H}\left(T^{*}(x), T^{*}(y)\right)$ for each $x, y \in H$.

Proof. Let $\mathfrak{F}$ denote the collection of all pairs $(D, T)$, where $T: D \rightarrow H, T(d) \in T^{*}(d) \forall d \in D$, $\overline{\text { and } d(T}(x), T(y)) \leq d_{H}\left(T^{*}(x), T^{*}(y)\right)$ for each $x, y \in D$.

Notice that $\mathfrak{F} \neq \emptyset$ since $\left(\left\{x_{0}\right\}, T\right) \in \mathfrak{F}$ for any choice of $x_{0} \in H$ and $T\left(x_{0}\right) \in T^{*}\left(x_{0}\right)$. Define an order relation on $\mathfrak{F}$ by setting $\left(D_{1}, T_{1}\right) \preceq\left(D_{2}, T_{2}\right) \Leftrightarrow D_{1} \subset D_{2}$ and $\left.T_{2}\right|_{D_{1}}=T_{1}$. Let $\left\{\left(D_{\alpha}, T_{\alpha}\right)\right\}$ be an increasing chain in $(\mathfrak{F}, \preceq)$. Then it follows that $\left(\cup_{\alpha} D_{\alpha}, T\right) \in \mathfrak{F}$ where $\left.T\right|_{D_{\alpha}}=T_{\alpha}$. By Zorn's Lemma, $(\mathfrak{F}, \preceq)$ has a maximal element, say $(D, T)$. Assume $D \neq H$ and select $x_{0} \in H \backslash D$. Set $\tilde{D}=D \cup\left\{x_{0}\right\}$ and consider the set

$$
J=\bigcap_{x \in D} B\left(T(x), d_{H}\left(T^{*}(x), T^{*}\left(x_{0}\right)\right)\right) \bigcap T^{*}\left(x_{0}\right) .
$$

Since $T^{*}\left(x_{0}\right) \in \mathcal{E}(H)$ for each $x \in H, J \neq \emptyset$ if and only if for each $x \in D \operatorname{dist}\left(T(x), T^{*}\left(x_{0}\right)\right) \leq$ $d_{H}\left(T^{*}(x), T^{*}\left(x_{0}\right)\right)$. Also, since $T^{*}\left(x_{0}\right)$ is a proximinal subset of $H$, the above is true if and only if for each $x \in D$,

$$
B\left(T(x), d_{H}\left(T^{*}(x), T^{*}\left(x_{0}\right)\right) \cap T^{*}\left(x_{0}\right) \neq \emptyset .\right.
$$

By the definition of Hausdorff distance $T^{*}(x) \subset N_{d_{H}\left(T^{*}(x), T^{*}\left(x_{0}\right)\right)+\varepsilon}\left(T^{*}\left(x_{0}\right)\right)$ for each $\varepsilon>0$.

However by assumption $T(x) \in T^{*}(x)$ so it must be the case that for each $\varepsilon>0$,

$$
B\left(T(x), d_{H}\left(T^{*}(x), T^{*}\left(x_{0}\right)\right)+\varepsilon\right) \cap T^{*}\left(x_{0}\right) \neq \emptyset .
$$

Since $T^{*}\left(x_{0}\right)$ is proximinal in $H$, this in turn implies

$$
B\left(T(x), d_{H}\left(T^{*}(x), T^{*}\left(x_{0}\right)\right)\right) \cap T^{*}\left(x_{0}\right) \neq \emptyset .
$$


Thus we conclude $J \neq \emptyset$. Choose $y_{0} \in J$ and define

$$
\tilde{T}(x)=\left\{\begin{array}{cc}
y_{0} & \text { if } x=x_{0} \\
T(x) & \text { if } x \in D
\end{array}\right.
$$

Since $d\left(\tilde{T}\left(x_{0}\right), \tilde{T}(x)\right)=d\left(y_{0}, T(x)\right) \leq d_{H}\left(T^{*}(x), T^{*}\left(x_{0}\right)\right)$ we conclude that $\left(D \cup\left\{x_{0}\right\}, \tilde{T}\right) \in \mathfrak{F}$, contradicting the maximality of $(D, T)$. Therefore $D=H$.

Looking in detail at this proof it comes obvious that the hyperconvexity of $H$ has not been used but in order to work with the images, hence the following corollary holds.

Corollary 9.2. The above theorem remains true if $T^{*}: M \rightarrow \mathcal{E}(H)$ with $M$ and $H$ any metric spaces and $\mathcal{E}(E)$ the class of bounded externally hyperconvex spaces of $H$ (assuming this class is nonempty).

The following corollary is a direct consequence of Theorem 9.1 combined with Theorem 6.1.

Corollary 9.3. Let $H$ be bounded and hyperconvex, and suppose $T^{*}: H \rightarrow \mathcal{E}(H)$ is nonexpansive. Then $T^{*}$ has a fixed point, that is, there exists $x \in H$ such that $x \in T^{*}(x)$.

The method of Theorem 9.1 also gives the following. In this theorem $\operatorname{Fix}\left(T^{*}\right)=\{x \in H: x \in$ $\left.T^{*}(x)\right\}$.

Theorem 9.4. Let $H$ be hyperconvex, let $T^{*}: H \rightarrow \mathcal{E}(H)$ be nonexpansive and suppose Fix $\left(T^{*}\right) \neq$ $\emptyset$. Then there exists a nonexpansive mapping $T: H \rightarrow H$ with $T(x) \in T^{*}(x)$ for each $x \in H$ and for which Fix $(T)=$ Fix $\left(T^{*}\right)$. In particular, Fix $\left(T^{*}\right)$ is hyperconvex.

Proof. Let $\mathfrak{F}$ denote the collection of all pairs $(D, T)$, where $D \supset \operatorname{Fix}\left(T^{*}\right), T: D \rightarrow H, T(d) \in$

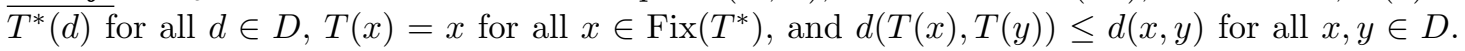
By assumption $\left(\operatorname{Fix}\left(T^{*}\right), I d\right) \in \mathfrak{F}$, so $\mathfrak{F} \neq \emptyset$. The argument is now a simple modification of the proof of Theorem 9.1. Define an order relation on $\mathfrak{F}$ by setting

$$
\left(D_{1}, T_{1}\right) \preceq\left(D_{2}, T_{2}\right) \Leftrightarrow D_{1} \subset D_{2} \text { and }\left.T_{2}\right|_{D_{1}}=T_{1} .
$$

Let $\left\{\left(D_{\alpha}, T_{\alpha}\right)\right\}$ be an increasing chain in $(\mathfrak{F}, \preceq)$. Then it follows that $\left(\cup_{\alpha} D_{\alpha}, T\right) \in \mathfrak{F}$ where $\left.T\right|_{D_{\alpha}}=T_{\alpha}$. By Zorn's Lemma, $(\mathfrak{F}, \preceq)$ has a maximal element, say $(D, T)$. Assume $D \neq H$ and select $x_{0} \in H \backslash D$. Set $\tilde{D}=D \cup\left\{x_{0}\right\}$ and consider the set

$$
J=\bigcap_{x \in D} B\left(T(x), d\left(x, x_{0}\right)\right) \bigcap T^{*}\left(x_{0}\right) .
$$

Since $T^{*}\left(x_{0}\right) \in \mathcal{E}(H)$ for each $x \in H, J \neq \emptyset$ if and only if for each $x \in D \operatorname{dist}\left(T(x), T^{*}\left(x_{0}\right)\right) \leq$ $d\left(x, x_{0}\right)$. Also, since $T^{*}\left(x_{0}\right)$ is a proximinal subset of $H$, the above is true if and only if for each $x \in D, B\left(T(x), d\left(x, x_{0}\right)\right) \cap T^{*}\left(x_{0}\right) \neq \emptyset$. Using the definition of Hausdorff distance and the fact that $T^{*}$ is nonexpansive, for each $\varepsilon>0$

$$
T^{*}(x) \subset N_{d_{H}\left(T^{*}(x), T^{*}\left(x_{0}\right)\right)+\varepsilon}\left(T^{*}\left(x_{0}\right)\right) \subset N_{d\left(x, x_{0}\right)+\varepsilon}\left(T^{*}\left(x_{0}\right)\right) .
$$

However by assumption $T(x) \in T^{*}(x)$ so it must be the case that for each $\varepsilon>0$,

$$
\left.B\left(T(x), d\left(x, x_{0}\right)\right)+\varepsilon\right) \cap T^{*}\left(x_{0}\right) \neq \emptyset .
$$

Since $T^{*}\left(x_{0}\right)$ is proximinal in $H$, this in turn implies $B\left(T(x) ; d\left(x, x_{0}\right)\right) \cap T^{*}\left(x_{0}\right) \neq \emptyset$. Thus we conclude $J \neq \emptyset$. Choose $y_{0} \in J$ and define

$$
\tilde{T}(x)=\left\{\begin{array}{cc}
y_{0} & \text { if } x=x_{0} \\
T(x) & \text { if } x \in D .
\end{array}\right.
$$


Since $d\left(\tilde{T}\left(x_{0}\right), \tilde{T}(x)\right)=d\left(y_{0}, T(x)\right) \leq d\left(x, x_{0}\right)$ we conclude that $\left(D \cup\left\{x_{0}\right\}, \tilde{T}\right) \in \mathfrak{F}$, contradicting the maximality of $(D, T)$. Therefore $D=H$. To conclude the proof, we need to show that $\operatorname{Fix}\left(T^{*}\right)$ is hyperconvex. This is a direct consequence of Theorem 6.1 applied to $T$.

Remark 9.5. Due to its importance in different branches of mathematics, selection problems have been widely studied along the last fifty years. The problem is usually as follows: given a certain multivalued mapping to be able to find an univalued selection of it with certain properties as, for instance, continuity or measurability. Theorem 9.1 is very surprising since it is not common at all to be able to guarantee that a nonexpansive multivalued mapping admits a nonexpansive selection, in fact this seems to be a quite characteristic fact from the hyperconvex geometry. Henceforth one of the most challenging open problems in hyperconvex metric spaces is whether this theorem may be improved or not in the sense that whether $\mathcal{E}(H)$ may be replaced by a wider class of subsets of $M$ or not. More specifically the most natural question at this moment is whether $\mathcal{W}(H)$, the class of nonempty bounded weakly externally hyperconvex subsets, could replace $\mathcal{E}(H)$ in Theorem 9.1. Counterexamples are not known for the case $\mathcal{H}(H)$. The reader will find a large collection of results and references on multivalued selection problems in the recent book [50].

We finish this section with two application of Theorem 9.1. First we show that the family of all bounded $\lambda$-lipschitzian functions of a hyperconvex space $M$ into itself is itself hyperconvex and second we will study a best approximation problem in hyperconvex spaces.

Let $f$ and $g$ be two bounded $\lambda$-lipschitzian functions of a hyperconvex space $M$ into itself, we define the distance between them in the usual way, that is, if $f, g: M \rightarrow M$, set

$$
d(f, g)=\sup _{x \in M} d(f(x), g(x)) .
$$

Theorem 9.6. Let $M$ be hyperconvex and for $\lambda>0$ let $\mathfrak{F}_{\lambda}$ denote the family of all bounded $\lambda$-lipschitzian functions of $M$ into $M$. Then $\mathfrak{F}_{\lambda}$ is itself a hyperconvex space.

Proof. Suppose $\left\{f_{\alpha}\right\} \subset \mathfrak{F}_{\lambda}$ and $\left\{r_{\alpha}\right\} \subset \mathbb{R}$ satisfy $d\left(f_{\alpha}, f_{\beta}\right) \leq r_{\alpha}+r_{\beta}$. Then for each $x \in M$ $\left.\overline{d\left(f_{\alpha}(x)\right.}, f_{\alpha}(x)\right) \leq r_{\alpha}+r_{\beta}$, so in view of the hyperconvexity of $M$

$$
J(x)=\bigcap_{\alpha} B\left(f_{\alpha}(x), r_{\alpha}\right) \neq \emptyset
$$

We show that $d_{H}(J(x), J(y)) \leq \lambda d(x, y)$ for each $x, y \in M$. To see this it clearly suffices to show that $J(x) \subset N_{\lambda d(x, y)}(J(y))$. for each $x, y \in M$. However if $z \in J(x)$ then for each $\alpha$

$$
\begin{aligned}
d\left(z, f_{\alpha}(y)\right) & \leq d\left(z, f_{\alpha}(x)\right)+d\left(f_{\alpha}(x), f_{\alpha}(y)\right) \\
& \leq d\left(z, f_{\alpha}(x)\right)+\lambda d(x, y) \\
& \leq r_{\alpha}+\lambda d(x, y) .
\end{aligned}
$$

Using Sine's Lemma (Lemma 4.10) we now have

$$
z \in \bigcap_{\alpha} B\left(f_{\alpha}(y), r_{\alpha}+\lambda d(x, y)\right)=N_{\lambda d(x, y)}(J(y))
$$

In view of Theorem 9.1 it is possible to select $f(x) \in J(x)$ for each $x \in M$ so that $f \in \mathfrak{F}_{\lambda}$. Since $f \in \bigcap_{\alpha} B\left(f_{\alpha}, r_{\alpha}\right), \mathfrak{F}_{\lambda}$ is hyperconvex.

This leads to the following. 
Corollary 9.7. Let $M$ be a bounded hyperconvex metric space and let $f \in \mathcal{F}_{1}$. Then the family

$$
R=\left\{r \in \mathcal{F}_{1}: r(M) \subset F i x(f)\right\}
$$

is a nonexpansive retract of $\mathcal{F}_{1}$.

Proof. The mapping $T_{f}: \mathcal{F}_{1} \rightarrow \mathcal{F}_{1}$ defined via the formula $T_{f}(g)=f \circ g$ is nonexpansive and has a nonempty fixed point set $\operatorname{Fix}\left(T_{f}\right)$ which is hyperconvex. Theorem 4.4 will then imply that $\operatorname{Fix}\left(T_{f}\right)$ is a nonexpansive retract of $\mathcal{F}_{1}$. But $r \in \operatorname{Fix}\left(T_{f}\right)$ if and only if $r \in R$.

One of the most important concepts in approximation theory is that one of metric projection. In this last part of the section we study the problem of finding nonexpansive selections of the metric projection. Let us introduce some definitions first. Recall that the concept of proximinality was introduced in Definition 3.7.

Definition 9.8. Let $M$ be a metric space and $A$ a proximinal subset of $M$, then the mapping $R: M \rightarrow 2^{A}$ defined as

$$
R(x)=B(x, \operatorname{dist}(x, A)) \cap A
$$

for every $x \in M$ is called the metric projection on $A$ (relative to $M$ ).

Notice that the proximinality of $A$ guarantees that $R(x) \neq \emptyset$ for all $x \in M$. We will also deal with the following concept.

Definition 9.9. A subset $A$ of a metric space $M$ is said to be a proximinal nonexpansive retract of $M$ if there exists a nonexpansive selection of the metric projection on $A$, i.e. if there exists a nonexpansive retraction $r: M \rightarrow A$ such that $r(x) \in B(x, \operatorname{dist}(x, A)) \cap A$ for each $x \in M$.

The first one in taking up the problem of characterizing proximinal nonexpansive retracts in hyperconvex metric spaces was Sine [56]. The following theorem was stated in [56] for admissible subsets, we adapt Sine's proof to externally hyperconvex subsets.

Theorem 9.10. Let $E$ be a nonempty externally hyperconvex subset of a metric space $H$. Then $E$ is a proximinal nonexpansive retract of $H$.

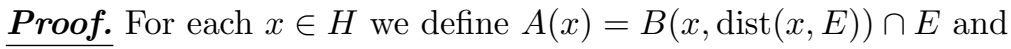

$$
C(x)=\bigcap\{A(y)+d(x, y): y \in H\} .
$$

Since $A(x)$ is one of the sets that define $C(x)$, the inclusion $C(x) \subseteq A(x)$ is clear. We will show that $C: H \rightarrow 2^{E}$ is a nonexpansive multivalued mapping which values are nonempty externally hyperconvex subsets of $H$. Due to the externally hyperconvexity of $E$ it will be enough to prove, in order to state the nonemptyness of $A(x)$, that

$$
B\left(y_{1}, \operatorname{dist}\left(y_{1}, E\right)+d\left(x, y_{1}\right)\right) \cap B\left(y_{2}, \operatorname{dist}\left(y_{2}, E\right)+d\left(x, y_{2}\right)\right) \neq \emptyset
$$

for each $y_{1}$ and $y_{2}$ in $H$. But $x$ belongs to both of these sets, so $C(x) \neq \emptyset$. Additionally, the externally hyperconvexity of $C(x)$ follows as an easy consequence of Lemma 5.3. Let us see now that $C$ is nonexpansive, for this pick $u$ and $v$ in $H$. We have to show that $C(u) \subseteq C(v)+d(u, v)$, or equivalently

$$
\bigcap_{z \in H}(A(z)+d(u, z)) \subseteq \bigcap_{z \in H}(A(z)+d(z, v)+d(u, v)),
$$

which is clear if one recalls that $d(u, z) \leq d(u, v)+d(v, z)$. Now the theorem follows as an application of Theorem 9.1. 
This problem was taken up again in [15] with some improvements of the above theorem. We state these results without proofs, which may be found in [15].

Theorem 9.11. A compact subset $E$ of a hyperconvex metric space $H$ is a proximinal nonexpansive retract of $H$ if and only if $E$ is weakly externally hyperconvex (relative to $H$ ).

The situation for the noncompact case has not been solved yet although the following theorem states a partial result that gets very close to completely remove the compact condition on Theorem 9.11 .

Theorem 9.12. Let $E$ be a weakly externally hyperconvex subset of a hyperconvex metric space $H$. Then given any $\varepsilon>0$ there is a nonexpansive retraction $R_{\varepsilon}$ of $H$ onto $E$ with the property that given any $u \in H \backslash E$ there exists $x \in H$ such that $d(u, x) \leq \varepsilon$ and $d\left(x, R_{\varepsilon}(x)\right)=\operatorname{dist}(x, E)$. Moreover if int $(E) \neq \emptyset$ then $R_{\varepsilon}$ may be chosen so that $R_{\varepsilon}(H \backslash E) \subset \partial E$.

Sine made used of Theorem 9.10 in order to obtain certain Ky Fan [17] type theorems for hyperconvex spaces. Even though Theorem 9.12 does not quite solve the "nonexpansive proximinal retract" problem it still enjoys enough good properties how to lead to improved new versions of Ky Fan type results given by Sine. We conclude this section with these fixed point results.

The first result we will see is a topological one, as those seen in Section 7, with boundary condition. In fact it extends Darbo-Sadovskii Theorem.

Theorem 9.13. Suppose $E$ is a bounded weakly externally hyperconvex subset of a hyperconvex space $H$ with nonempty interior, and let $T: E \rightarrow H$ be a uniformly continuous condensing mapping for which $T(\partial E) \subset E$. Then $T$ has a fixed point.

Proof. Let $\varepsilon>0$ and choose $\varepsilon^{\prime} \leq \varepsilon$ so that $d(u, v) \leq \varepsilon^{\prime} \Rightarrow d(T(u), T(v)) \leq \varepsilon$. Now let $R_{\varepsilon^{\prime}}$ be the nonexpansive retraction assured by Theorem 9.12. It is easy to see that the mapping $R_{\varepsilon^{\prime}} \circ T: E \rightarrow E$ is condensing, and since $E$ is hyperconvex $R_{\varepsilon^{\prime}} \circ T$ has a fixed point, say $x_{\varepsilon} \in$ $D$. If $T\left(x_{\varepsilon}\right) \in E$, then $R_{\varepsilon^{\prime}} \circ T\left(x_{\varepsilon}\right)=T\left(x_{\varepsilon}\right)=x_{\varepsilon}$. If $T\left(x_{\varepsilon}\right) \notin E$, then there exists $y \in \partial E$ such that $d\left(x_{\varepsilon}, y\right) \leq \varepsilon^{\prime}$. In this case (since $T(y) \in E$ ) we have

$$
\begin{aligned}
d(y, T(y)) & \leq d\left(y, x_{\varepsilon}\right)+d\left(x_{\varepsilon}, T(y)\right) \\
& \leq \varepsilon+d\left(R_{\varepsilon^{\prime}} \circ T\left(x_{\varepsilon}\right), R_{\varepsilon^{\prime}} \circ T(y)\right) \\
& \leq \varepsilon+d\left(T\left(x_{\varepsilon}\right), T(y)\right) \\
& \leq 2 \varepsilon .
\end{aligned}
$$

This proves that inf $\{d(y, T(y)): y \in E\}=0$. Since $T$ is condensing it easily follows that $T$ has a fixed point in $D$.

The following is an easy consequence of Theorem 9.12.

Theorem 9.14. Suppose $E$ is a bounded weakly externally hyperconvex subset of a hyperconvex metric space $H$, and suppose $T: E \rightarrow H$ is a nonexpansive mapping for which $T(\partial E) \subset E$. Then $T$ has a fixed point.

Proof. Let $R$ be a nonexpansive retraction of $H$ onto $E$ for which $R(H \backslash E) \subseteq \partial E$. Then

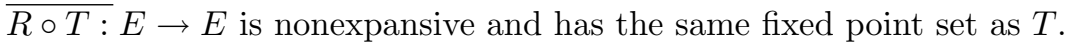

Another consequence of Theorem 9.12 is the following theorem. 
Theorem 9.15. Suppose $E$ is a bounded weakly externally hyperconvex subset of a hyperconvex metric space $H$, and let $T: E \rightarrow H$ be a condensing mapping for which $T(\partial E) \subset E$. Then $T$ has a fixed point.

Finally, since compact hyperconvex spaces have the fixed point property for continuous mappings (e.g., [31, 47]), Theorem 9.11 yields Ky Fan's approximation principle for compact weakly externally hyperconvex sets.

Theorem 9.16. Let $E$ and $H$ satisfy the assumptions of Theorem 9.11 and suppose $T: E \rightarrow H$ is a continuous mapping. Then there exists $x \in E$ such that

$$
d(x, T(x))=\inf \{d(y, T(x)): y \in D\} .
$$

Proof. Let $R$ be the retraction given by Theorem 9.11, then $R \circ T: E \rightarrow E$ is a continuous

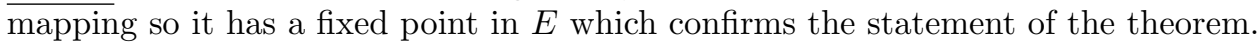

Ky Fan's fixed point theorem for hyperconvex metric space is henceforth stated in the following way.

Corollary 9.17. Let $E$ and $H$ satisfy the assumptions of Theorem 9.11 and suppose $T: E \rightarrow H$ is a continuous mapping such that $T(\partial E) \subseteq E$ (if interior of $E$ is empty, assume $T(E) \subseteq E$ ). Then $T$ has a fixed point in $E$.

Proof. It is easy to see that the fixed point of $R \circ T$ in the theorem must be a fixed point of $T$ $\overline{\text { under the additional boundary condition of the statement of the corollary. }}$

\section{The KKM TheOrY In Hyperconvex spaCeS}

Among the results equivalent to the Brouwer's fixed point theorem, the Theorem of KnasterKuratowski-Mazurkiewicz (in short KKM) occupies a special place. Historically Brouwer's fixed point theorem failed to impose itself in the metric setting in comparison with Kirk's fixed point theorem. In our opinion, this is due to the fact that the first theorem depends heavily on the convex structure of the set while the second one depends on a set theoretical kind of convexity. Since hyperconvex metric spaces exhibit some kind of convexity, it was natural to investigate Brouwer's fixed point theorem in this setting. This problem was first studied by the second author in [31] and later by others in [41,47]. This section is devoted to present some of the results appeared in these three works. The literature on KKM principle is quite large, the reader will find a more precise and exhaustive treatment of it in $[22,59]$ where KKM principle is mainly developed in topological and nonlinear settings.

Let $H$ be a metric space. A subset $A \subset H$ is called finitely closed if for every $x_{1}, x_{2}, \ldots, x_{n} \in H$, the set $\operatorname{cov}\left(\left\{x_{i}\right\}\right) \cap A$ is closed. If $A$ is closed then obviously it is also finitely closed. Recall that a family $\left\{A_{\alpha}\right\}_{\alpha \in \Gamma}$ in $2^{H}$ is said to have the finite intersection property if the intersection of each finite subfamily is not empty.

Definition 10.1. Let $H$ be a metric space and $X \subset H$. A multivalued mapping $G: X \rightarrow 2^{H}$ is called a Knaster-Kuratowski-Mazurkiewicz map (in short KKM-map) if

$$
\operatorname{cov}\left(\left\{x_{1}, \ldots, x_{n}\right\}\right) \subset \bigcup_{1 \leq i \leq n} G\left(x_{i}\right)
$$

for any $x_{1}, \ldots, x_{n} \in X$. 
We have the following result:

Theorem 10.2. (KKM-maps principle) Let $H$ be a hyperconvex metric space, and $X$ be a nonempty subset of $H$. Let $G: X \rightarrow 2^{H}$ be a KKM-map such that each $G(x)$ is finitely closed. Then the family $\{G(x) ; x \in X\}$ has the finite intersection property.

Proof. Assume not, i.e. there exist $x_{1}, \ldots, x_{n} \in X$ such that $\bigcap_{i=1}^{i=n} G\left(x_{i}\right)=\emptyset$. Set $L=\operatorname{cov}\left(\left\{x_{i}\right\}\right)$ in $H$. Consider the hyperconvex Banach space $l_{\infty}(H)$ and set $H_{\infty}=\operatorname{cov}(H)$ in $l_{\infty}(H)$. Let $C=\operatorname{conv}\left(x_{i}\right)$ in $H_{\infty}$. Here we consider the linear convex hull. By Theorem 4.4, there exists a nonexpansive retraction $r: H_{\infty} \rightarrow H$. Note that $r(C) \subset L$. Our assumptions imply that $L \cap G\left(x_{i}\right)$ is closed for every $i=1,2, \ldots, n$. Since $\bigcap G\left(x_{i}\right) \bigcap L=\emptyset$ then, for every $c \in C$, there exists $i_{0}$ such that $r(c)$ does not belong to $L \cap G\left(x_{i_{0}}\right)$. Hence $\operatorname{dist}\left(r(c), L \cap G\left(x_{i_{0}}\right)\right)>0$ because $L \cap G\left(x_{i_{0}}\right)$ is closed. Therefore, the function

$$
\alpha(c)=\sum_{i=1}^{i=n} \operatorname{dist}\left(r(c), L \cap G\left(x_{i}\right)\right)
$$

is not zero for any $c \in C$. Define the map $F: C \rightarrow C$ by

$$
F(c)=\frac{1}{\alpha(c)} \sum_{i=1}^{i=n} \operatorname{dist}\left(r(c), L \cap G\left(x_{i}\right)\right) x_{i} .
$$

Clearly, $F$ is a continuous map. Since $C$ is compact, then Brouwer's theorem implies the existence of a fixed point $c_{0}$ of $F$, i.e. $F\left(c_{0}\right)=c_{0}$. Set $I=\left\{i\right.$; $\left.\operatorname{dist}\left(r\left(c_{0}\right), L \cap G\left(x_{i}\right)\right) \neq 0\right\}$. Clearly we have

$$
c_{0}=\frac{1}{\alpha\left(c_{0}\right)} \sum_{i \in I} \operatorname{dist}\left(r\left(c_{0}\right), L \cap G\left(x_{i}\right)\right) x_{i} .
$$

Therefore, $r\left(c_{0}\right) \notin \bigcup_{i \in I} G\left(x_{i}\right)$ and $r\left(c_{0}\right) \in \operatorname{cov}\left(\left\{x_{i} ; i \in I\right\}\right)$, contradicting the assumption $\operatorname{cov}\left(\left\{x_{i} ; i \in\right.\right.$ $I\} \subset \cup_{i \in I} G\left(x_{i}\right)$. The proof of Theorem 10.2 is therefore complete.

As an immediate consequence, we obtain the following theorem.

Theorem 10.3. Let $H$ be a hyperconvex metric space and $X \subset H$ be a nonempty subset. Let $G: X \rightarrow 2^{H}$ be a KKM-map such that $G(x)$ is closed for any $x \in X$ and $G\left(x_{0}\right)$ is compact for some $x_{0} \in X$. Then we have

$$
\bigcap_{x \in X} G(x) \neq \emptyset
$$

Notice that the compactness assumption of $G\left(x_{0}\right)$ may be a stronger one. We can still reach the conclusion if one involves an auxiliary multivalued map and a suitable topology on $H$ (such as the ball topology for example).

Theorem 10.4. Let $H$ be a hyperconvex metric space and $X \subset H$ be a nonempty subset. Let $G: X \rightarrow 2^{H}$ be a KKM-map. Assume there exists a multivalued map $K: X \rightarrow 2^{H}$ such that $G(x) \subset K(x)$ for every $x \in X$ and

$$
\bigcap_{x \in X} K(x)=\bigcap_{x \in X} G(x) .
$$


If there exists a topology $\tau$ on $H$ such that each $K(x)$ is compact for $\tau$, then

$$
\bigcap_{x \in X} G(x) \neq \emptyset \text {. }
$$

The proof is obvious.

The concept of KKM mapping was generalized in [41] in the following way.

Definition 10.5. Let $H$ be a metric space and $X \subseteq H$. A multivalued mapping $G: X \rightarrow 2^{H} \backslash\{\emptyset\}$ is called a generalized metric KKM mapping (GMKKM) if for each finite set $\left\{x_{1}, \ldots, x_{n}\right\} \subseteq X$, there exists a set $\left\{y_{1}, \ldots, y_{n}\right\}$ of points of $H$, not necessarily all different, such that for each subset $\left\{y_{i_{1}}, \ldots, y_{i_{k}}\right\}$ of $\left\{y_{1}, \ldots, y_{n}\right\}$ we have $\operatorname{cov}\left\{y_{i_{j}}: j=1, \ldots k\right\} \subseteq \cup_{j=1}^{k} G\left(x_{i_{j}}\right)$.

It is easy to check that KKM mappings are generalized KKM mappings while the converse is not true, the interested reader may consult $[41,59]$ for more about this topic. The following theorem is the extension of Theorem 10.2 where generalized metric KKM mappings substitute KKM mappings. The proof, although more complicated, follows similar ideas to those in the proof of Theorem 10.2 and we will omit it.

Theorem 10.6. (Generalized metric KKM principle) Let $H$ be a hyperconvex metric space, and $X$ be a nonempty subset of $H$. Suppose $G: X \rightarrow 2^{H} \backslash\{\emptyset\}$ has finitely closed values. Then the family $\{G(x): x \in X\}$ has the finite intersection property it and only if the mapping $G$ is a generalized metric KKM mapping.

Kirk at al. exhibit a large number of consequences of this theorem in [41] (see also [40, 47, 59]). These consequences have to do with Minimax inequalities, fixed point theorems for multivalued mappings, saddle points, and Nash equilibria. The following theorem is among these consequences. Note that this theorem is a multivalued version of Ky Fan's approximation principle already seen in the previous section.

Theorem 10.7. Let $H$ be a hyperconvex space and $A$ a nonempty admissible compact subset of $H$. Suppose $T: A \rightarrow \mathcal{A}(H)$ is a multivalued continuous mapping. Then there exists $x_{0} \in A$ such that

$$
\operatorname{dist}\left(x_{0}, T\left(x_{0}\right)\right)=\inf _{x \in A} \operatorname{dist}\left(x, T\left(x_{0}\right)\right) .
$$

Proof. Define the mapping $G: A \rightarrow 2^{H} \backslash\{\emptyset\}$ by $G(x)=\{y \in A: \operatorname{dist}(y, T(y)) \leq \operatorname{dist}(x, T(y))\}$ for each $x \in A$. As $T$ is continuous, $G(x)$ is closed and nonempty for each $x \in A$. We want to prove that $G$ is a KKM mapping. Suppose it is not, then there exists a nonempty and finite subset $\left\{x_{1}, \ldots, x_{n}\right\}$ and $y \in \operatorname{cov}\left(\left\{x_{i}: i=1, \ldots, n\right\}\right)$ such that $\operatorname{dist}\left(x_{i}, T(y)\right)<\operatorname{dist}(y, T(y))$ for $i=1, \ldots, n$. Let $\varepsilon>0$ be such that $\operatorname{dist}\left(x_{i}, T(y)\right) \leq \operatorname{dist}(y, T(y))-\varepsilon$ for $i=1, \ldots, n$. Let $r=\operatorname{dist}(y, T(y))-\varepsilon$. Then $x_{i} \in T(y)+r$ for $i=1, \ldots, n$. From Lemma $4.10 T(y)+r \in \mathcal{A}(H)$, thus $\operatorname{cov}\left\{x_{1}, \ldots, x_{n}\right\} \subseteq$ $T(y)+r$. This in turn implies $y \in T(y)+r$ and hence $\operatorname{dist}(y, T(y)) \leq r=\operatorname{dist}(y, T(y))-\varepsilon$, which is not possible by assumption. Therefore $G$ must be a KKM mapping.

Note that $\cap_{x \in A} G(x) \neq \emptyset$ since $X$ is compact. Take $x_{0} \in \cap_{x \in A} G(x)$. Then it is clear that $\operatorname{dist}\left(x_{0}, T\left(x_{0}\right)\right) \leq \operatorname{dist}\left(x, T\left(x_{0}\right)\right)$ for all $x \in A$, which completes the proof of the theorem.

\section{LAMBDA-HYPERCONVEXITY}

Since the beginning it was known that the Hilbert space $\ell_{2}$ fails hyperconvexity. By studying this case closely, the second author, Knaust, Nguyen and O'Neill [34] introduced a property very 
similar to hyperconvexity, called $\lambda$-hyperconvexity. The idea is to expand the radius of the given balls by a uniform factor. For example, every pairwise intersecting collection of balls in $\ell_{2}$ has non-empty intersection if the radius of the balls are increased by the factor $\sqrt{2}$. In light of this, the following definition becomes natural.

Definition 11.1. Let $M$ be a metric space and let $\lambda \geq 1$. We say that the metric space $M$ is $\lambda$-hyperconvex if for every non-empty admissible set $A \in \mathcal{A}(M)$, for any family of closed balls $\left\{B\left(x_{\alpha}, r_{\alpha}\right)\right\}_{\alpha \in \Lambda}$, centered at $x_{\alpha} \in A$ for $\alpha \in \Lambda$, the condition

$$
d\left(x_{\alpha}, x_{\beta}\right) \leq r_{\alpha}+r_{\beta} \text { for every } \alpha, \beta \in \Lambda,
$$

implies

$$
A \cap\left(\bigcap_{\alpha \in \Lambda} B\left(x_{\alpha}, \lambda r_{\alpha}\right)\right) \neq \emptyset .
$$

Let $\lambda(M)$ be the infimum of all constants $\lambda$ such that $M$ is $\lambda$-hyperconvex, and say that $\lambda(M)$ is exact if $M$ is $\lambda(M)$-hyperconvex.

Grübaum [20] and other authors have studied a similar property not involving the underlying admissible set $A$. But its introduction becomes essential when we try to connect this concept to the fixed point property via the normal structure property.

Let us recall Grünbaum's definition: For a metric space $M$, let the expansion constant $E(M)$ be the infimum of all constants $\mu$ such that the following holds: Whenever a collection $\left\{B\left(x_{\alpha}, r_{\alpha}\right)\right.$ : $\alpha \in \Lambda$ \} intersects pairwise, then

$$
\bigcap_{\alpha \in \Lambda} B\left(x_{\alpha}, \mu \cdot r_{\alpha}\right) \neq \emptyset
$$

We say $E(M)$ is exact, if the condition is even satisfied for $\mu=E(M)$.

Trivially, $E(M) \leq \Lambda(M)$ holds in metrically convex spaces (see Definition 2.3). On the other hand, if $M$ is a two element metric space, then $E(M)=1$, while $\Lambda(M)=2$, so both concepts do not coincide in general.

Let us first summarize some basic properties of $\lambda$-hyperconvex metric spaces, some of which are trivial, while the others can be easily derived from corresponding results about expansion constants:

Theorem 11.2. Let $M$ be a metric space.

1. $M$ is hyperconvex if and only if it is 1-hyperconvex.

2. Every $\lambda$-hyperconvex metric space is complete.

3. Reflexive Banach spaces and dual Banach spaces are 2-hyperconvex.

4. There is a subspace $X$ of $\ell_{1}$ which fails to be 2-hyperconvex.

5. Hilbert space is $\sqrt{2}$-hyperconvex.

We will finish this section, and hence this chapter on hyperconvexity, by studying the connection between $\lambda$-hyperconvexity and the fixed point property. The theorem we will finish with is based on a metric generalization of Kirk's fixed point theorem established in [30]. In order to state this generalization we need to know what a uniform normal structure on a metric space is.

Let $M$ be a metric space and $\mathcal{F}$ a family of subsets of $M$. Then we say that $\mathcal{F}$ defines a convexity structure on $M$ if it contains the closed balls and is stable by intersection. For instance $\mathcal{A}(M)$, the class of the admissible subsets of $M$, defines a convexity structure on any metric 
space $M$. We say that $\mathcal{F}$ is a uniform normal structure on $M$ if there exists $c<1$ such that $R(A) \leq c \cdot \operatorname{diam}(A)$ for every $A \in \mathcal{F}$ with $\operatorname{diam}(A)>0$, where $R(A)$ and $\operatorname{diam}(A)$ are, respectively, the Chebyshev radius and diameter of $A$ defined in Section 3.

Now we may state the generalization of Kirk's fixed point theorem.

Theorem 11.3. Let $M$ be a bounded complete metric space. If $M$ has a uniform normal structure then it has the fixed point property for nonexpansive mappings.

The connection between $\lambda$-hyperconvexity and the fixed point property is giving by the following theorem.

Theorem 11.4. Let $M$ be a bounded $\lambda$-hyperconvex space. If $\lambda<2$, then any nonexpansive mapping $T: M \rightarrow M$ has a fixed point.

Proof. Let $M$ be a bounded $\lambda$-hyperconvex space with $\lambda<2$. Theorem 11.2 assures that $M$ is complete, so from the previous theorem it suffices to prove that $M$ has a uniform normal structure. The family $\mathcal{A}(M)$ defines a convexity structure on $M$, we will show that $\mathcal{A}(M)$ is actually a uniform normal structure on $M$. Let $A \in \mathcal{A}(M)$ with $\operatorname{diam}(A)>0$. For each $x \in A$ let $B\left(x, r_{x}\right)$ denote the ball centered at $x$ with constant radius $r_{x}=\frac{1}{2} \operatorname{diam}(A)$. Then $d(x, y) \leq r_{x}+r_{y}$ for every $x, y \in A$. Since $M$ is $\lambda$-hyperconvex we can find

$$
x_{0} \in A \cap\left(\cap_{x \in A} B\left(x, \lambda r_{x}\right)\right) .
$$

Thus we have $d\left(x, x_{0}\right) \leq \frac{1}{2} \lambda \operatorname{diam}(A)$ for every $x \in A$. It follows that $R(A) \leq \frac{1}{2} \lambda \operatorname{diam}(A)$. Finally since $\frac{1}{2} \lambda<1$ we obtain that $\mathcal{A}(M)$ is a uniform normal structure on $M$, and hence the theorem is proved.

\section{REFERENCES}

[1] N. Aronszajn, and P. Panitchpakdi, Extension of uniformly continuous transformations and hyperconvex metric spaces, Pacific J. Math. 6 (1956), 405-439.

[2] J. M. Ayerbe, T. Dominguez, and G. Lpez, Measures of Noncompactness in Metric Fixed Point Theory, Operator Theory: Advances and Applications, 99, Birkhuser Verlag, Basilea 1997.

[3] J. B. Baillon, Nonexpansive mappings and hyperconvex spaces, Contemp. Math. 72 (1988), 11-19.

[4] D. Bugajewski, and R. Espínola, Remarks on some fixed point theorems for hyperconvex metric spaces and absolute retracts, Lecture Note in Pure and Applied Mathematic 213, New-York Marcel-Dekker 2000 (85-92).

[5] D. Bugajewski, and E. Grzelaczyk, A fixed point theorem in hyperconvex spaces, Arch. Math. 75 (2000), 395-400.

[6] D. Cartwright, Extension of positive operators between Banach lattices, Mem. Amer. Math. Soc. V. 3 164 (1975), 1-48.

[7] F. Cianciaruso, and E. De Pascale, Discovering the algebraic structure on the metric injective envelope of real Banach space, Topology and its Applications 78 (1997), 285-292.

[8] F. Cianciaruso, and E. De Pascale, The Hausdorff measure of non hyperconvexity, Atti Sem. Mat. Fis. Univ. Modena, XLVII (1999), 261-267.

[9] H. B. Cohen, Injective envelopes of Banach spaces, Bull. Amer. Math. Soc. 70 (1964), $723-726$.

[10] H. B. Cohen, and H. E. Lacey, On injective envelopes of Banach spaces, J. Funct. Anal. 4 (1969), 11-30.

[11] G. Darbo, Punti uniti in trasformazioni a condominio non compatto, Rend. Sem. Mat. Univ. Padova 24 (1955), 84-92.

[12] T. Domjnguez (edit.), Recent Advances on Metric Fixed Point Theory, Universidad de Sevilla, Sevilla, 1996.

[13] J. Dugundji, and A. Granas, Fixed Point Theory, Polish Scientific Publisher, Warszawa, 1982.

[14] R. Espinola, Darbo-Sadovski's theorem in hyperconvex metric spaces, Supplemento ai Rendiconti del Circolo Matematico di Palermo 40 (1996), 130-139. 
[15] R. Espinola, W. A. Kirk, and G. Lpez, Nonexpansive retractions in hyperconvex spaces, J. Math. Anal. Appl. 251 (2000), 557-570.

[16] R. Espinola, and G. Lpez, Ultimately compact operators in hyperconvex metric spaces, Nonlinear Analysis and Convex Analysis, Eds: W. Takahasi and T. Tamaka, World Scientific Publishing, 1999, 142-149.

[17] K. Fan, Extensions of two fixed point theorems of F. E. Browder, Math. Z. 112 (1969), 234-240.

[18] K. Goebel, and W. A. Kirk, Topics on Metric Fixed Point Theory, Cambridge Univ. Press, Cambridge, 1990.

[19] D. B. Goodner, Projections in normed linear spaces, Trans. Amer. Math. Soc. 69 (1950), 89-108.

[20] B. Grnbaum, On some covering and intersection properties in Minkowski spaces, Pacific J. Math. 27 (1959), 487-494.

[21] H. Herrlich, On hyperconvex hulls of metric spaces, Topology and its Applications 46 (1992), 13-21.

[22] C. Horvath, Contractibility and generalized convexity, J. Math. Anal. Appl. 156 (1991), 341-357.

[23] O. Hustad, Intersection properties of balls in complex Banach spaces whose duals are $L^{1}$ spaces, Acta Math. 132 (1974), 283-213.

[24] J. R. Isbell, Six theorems about injective metric spaces, Comment. Math. Helvetic 39 (1964), 65-76.

[25] J. R. Isbell, Injective envelopes of Banach spaces are rigidly attached, Bull. Amer. Math. Soc. 70 (1964), 727-729.

[26] J. R. Isbell, Three remarks on injective envelopes of Banach spaces, J. Math. Anal. Appl. 27 (1969), 516-518.

[27] E. Jawhari, D. Misane, and M. Pouzet, Retracts: graphs and ordered sets from the metric point of view, Contemp. Math. 57 (1986), 175-226.

[28] J. Jaworowski, W. A. Kirk, and S. Park, Antipodal Points and Fixed Points, Notes of the Series of lectures held at the Seoul National Univesity, 1995.

[29] J. L. Kelley, Banach spaces with the extension property, Trans. Amer. Math. Soc. 72 (1952), 323-326.

[30] M. A. Khamsi, On metric spaces with uniform normal structure, Proc. Amer. Math. Soc. 106 (1989), 723-726.

[31] M. A. Khamsi, KKM and Ky Fan Theorems in hyperconvex metric spaces, J. Math. Anal. Appl. 204 (1996), 298-306.

[32] M. A. Khamsi, One-local retract and common fixed point for commuting mappings in metric spaces, Nonlinear Anal. 27 (1996), 1307-1313.

[33] M. A. Khamsi, W. A. Kirk, and C. Martinez Y ez, Fixed point and selection theorems in hyperconvex spaces, Proc. Amer. Math. Soc. 128 (2000), 3275-3283.

[34] M. A. Khamsi, H. Knaust, N. T. Nguyen, and M. D. O'Neill, Lambda-hyperconvexity in metric spaces Nonlinear Anal. 43 (2000), 21-31.

[35] M. A. Khamsi, M. Lin, and R. C. Sine, On the fixed points of commuting nonexpansive maps in hyperconvex spaces, J. Math. Anal. Appl. 168 (1992), 372-380.

[36] M. A. Khamsi, and S. Reich, Nonexpansive mappings and semigroups in hyperconvex spaces, Math. Japonica 3 (1990), n. 11, 467-471.

[37] W. A. Kirk, A fixed point theorem for mappings which do not increase distances, Amer. Math. Monthly, 72 (1965), 1004-1006.

[38] W. A. Kirk, An abstract fixed point theorem for nonexpansive mappings, Proc. Amer. Math. Soc. 82 (1981), 640-642.

[39] W. A. Kirk, Hyperconvexity of $\mathbb{R}$-trees, Fundamenta Mathematicae 156 (1998), 67-72.

[40] W. A. Kirk, and S. S. Shin, Fixed point theorems in hyperconvex spaces, Houston J. Math. 23 (1997), 175-187.

[41] W. A. Kirk, B. Sims, and G. Xian-Zhi Yuan, The Knaster, Kuratowski and Mazurkiewicz theory in hyperconvex metric spaces and some of its applications, Nonlinear Anal. 39 (2000), n. 5, 611-627.

[42] J. L. Lacey, The Isometric Theory of Classical Banach Spaces, Springer Verlag, Berlin, Heidelberg, New York, 1974.

[43] Å. Lima, Intersection properties of balls and subspaces in Banach spaces, Trans. Amer. Math. Soc. 227 (1977), 1-62.

[44] M. Lin, and R. C. Sine, Retractions on the fixed point set of semigroups of nonexpansive maps in hyperconvex spaces, Nonlinear Anal. 15 (1990), 943-954.

[45] J. Lindenstrauss, Extension of compact operators, Mem. Amer. Math. Soc. 48 (1964), 1-112.

[46] L. Nachbin, A theorem of the Hahn-Banach type, Trans. Amer. Math. Soc. 68 (1950), 28-46.

[47] S. Park, Fixed point theorems in hyperconvex metric spaces, Nonlinear Anal. 37 (1998), n. 4, 467-472.

[48] S. Park, The Schauder type and other fixed point theorems in hyperconvex spaces, Nonlinear Analysis Forum (1998), n. 3, 1-12.

[49] J. P. Penot, Fixed point theorems without convexity, Bull. Soc. Math. France Mmoire 60 (1979), 129-152.

[50] D. Repovš, and P. V. Semenov, Continuous Selections of Multivalued Mappings, Kluwer Academic Publishers, Dordrecht, 1998. 
[51] N. V. Rao, The metric injective hulls of normed spaces, Topology and its Applications 46 (1992), 13-21.

[52] B. N. Sadovskii, A fixed point principle, Functional Analysis and Applications 1 (1967), 151-153.

[53] B. N. Sadovskii, Limit-compact and condensing operators, Russ. Math. Surveys 27 (1972), 85-155.

[54] R. C. Sine, On linear contraction semigroups in sup norm spaces, Nonlinear Anal. 3 (1979), 885-890.

[55] R. C. Sine, Hyperconvexity and approximate fixed points, Nonlinear Anal. 13 (1989), 863-869.

[56] R. C. Sine, Hyperconvexity and nonexpansive multifunctions, Trans. Amer. Math. Soc. 315 (1989), 755-767.

[57] P. Soardi, Existence of fixed points for nonexpansive mappings in certain Banach lattices, Proc. Amer. Math. Soc. 73 (1979), 25-29.

[58] S. Szufla, On the application of measure of noncompactness to existence theorems, Rend. Sem. Math. Univ. Padova 75 (1986), 1-14.

[59] G. Xian-Zhi Yuan, KKM Theory and Applications in Nonlinear Analysis, Marcel Dekker, Inc., New York 1999.

Remark. This paper appeared first in "Handbook of Metric Fixed Point Theory", by W.A. Kirk and B. Sims, published by Kluwer Academic Publishers in June 2001. 